

\title{
Dress induction and the Burnside quotient Green ring
}

\author{
Ian Hambleton, Laurence R. Taylor and Bruce Williams
}

\begin{abstract}
We define and study the Burnside quotient Green ring of a Mackey functor, introduced in our 1990 MSRI preprint. Some refinements of Dress induction theory are presented, together with applications to computation results for $K$-theory and $L$-theory of finite and infinite groups.
\end{abstract}

\section{Introduction}

Induction theory began with Artin and Brauer's work in representation theory, was continued by Swan [1970] and Lam [1968] for $K$-theory, and was put in its most abstract and elegant setting by Green [1971] and Dress [1973; 1975]. The theory sets up a convenient framework for computing the value of a Mackey functor on some finite group $G$, given suitable generation results for a Green ring which acts on the Mackey functor. (See [tom Dieck 1987; Lindner 1976; Thévenaz 1990; Thévenaz and Webb 1995] for some subsequent developments.)

The main examples in this theory are (i) the Swan Green ring $S W(G, \mathbf{Z})$ [Swan 1970], which leads to the Brauer-Berman-Witt induction theorem for representations of finite groups, and computation results for Quillen $K$-theory $K_{n}(R G)$, and (ii) the Dress Green ring $G U(G, \mathbf{Z})$ [Dress 1975], which leads to computation results for the oriented surgery obstruction groups $L_{n}(\mathbf{Z G )}$ of Wall [1976].

In Section 2 we define the Burnside quotient Green ring $\mathscr{A}_{\mathcal{M}}$ for a Mackey functor $\mathcal{M}: \mathscr{D}(G) \rightarrow \mathscr{A} b$, where $\mathscr{D}(G)$ denotes the category of finite $G$-sets, and $\mathscr{A} b$ the category of abelian groups. This Green ring $\mathscr{A}_{\mathcal{M}}$ is the smallest quotient of the Burnside ring which is a Green ring, and still acts on the Mackey functor. As defined, it has many convenient naturality properties, and generation results for $A_{M}$ will lead as usual to computation results for $\mathcal{M}$. We define the concept of a Dress generating set $X$ for a Green ring in Definition 3.5. The main result (see Theorem 3.6) is:

MSC2000: primary 20C15; secondary 18F25, 57R67, 19A22.

Keywords: Dress induction, Mackey functors, surgery obstruction groups.

Research partially supported by NSERC Discovery Grant A4000 and the NSF.. 
Theorem A. A finite $G$-set $X$ is a Dress generating set for a Green ring $\mathscr{G}$ if and only if it is a Dress generating set for the Burnside quotient Green ring Acg.

The naturality of the Burnside quotient Green rings can now be used to obtain computability results for sub- or quotient Mackey functors (see Theorem 3.8). We also point out a useful refinement of Dress induction in Theorem 3.10. We use the Burnside quotient Green ring in Section 5 to study additive functors out of the categories $R G$-Morita defined in our paper of 1990 , henceforth abbreviated [HTW 1990]; here $R$ is a commutative ring with unit. The main examples of such functors include $K$-theory, Hochschild homology and cyclic homology [HTW 1990, 1.A.12]. We define a bifunctor $d: \mathscr{D}(G) \rightarrow R G$-Morita in 5.4 and prove the following computability result (see Theorem 5.5):

Theorem B. Any additive functor $F: R G$-Morita $\rightarrow$ Ab gives a Mackey functor on $\mathscr{D}(G)$ by composition with $d: \mathscr{D}(G) \rightarrow R G$-Morita. Any such Mackey functor is hyperelementary computable.

This is a refinement of [Oliver 1988, 11.2], and Theorem A provides the key new ingredient in the proof. The extra generality is useful for studying functors such as the Dade group and the units in the Burnside ring [Bouc 2007; Bouc 2008].

The Burnside quotient Green ring has been applied to study the permutation representations of finite groups in [Hambleton and Taylor 1999], free actions of finite groups on products of spheres in [Hambleton 2006], and to the computation of Bass nilgroups in [Hambleton and Lück 2007]. This theory was surveyed and used in [Hambleton and Taylor 2000]. Our results also apply to the computation of $K$ and $L$-theory for infinite groups, based on an idea of Farrell and Hsiang [1981].

We introduce Mackey prefunctors and pseudo-Mackey functors in Section 6. A Mackey prefunctor is a just prebifunctor $\mathscr{D}(G) \rightarrow \mathscr{A} b$, and a pseudo-Mackey functor is a Mackey prefunctor which admits a finite filtration by Mackey functors. Such structures have been observed in a number of different contexts: the main examples include the higher Whitehead groups $\mathrm{Wh}_{n}(\mathbf{Z G})$, and the structure set of a compact manifold in surgery theory [Wall 1999, Chapter 9].

It turns out that the general scheme of Dress induction theory can be extended to pseudo-Mackey functors as well. In Section 7, we combine this idea with the Burnside quotient Green ring to study additive functors out of the category $(R G, \omega)$-Morita [HTW 1990, 1C]. We have the corresponding computability result (see Theorem 7.2):

Theorem C. Let $F:(R G, \omega)$-Morita $\rightarrow A A b$ be an additive functor. Then the composite $\mathcal{M}=F \circ d: \mathscr{D}(G) \rightarrow \mathscr{A} b$ is a Mackey prefunctor. Moreover:

(i) The 2-adic completion of $\mathcal{M}$ is 2-hyperelementary computable.

(ii) If $\mathcal{M}$ is a Mackey functor, then $\mathcal{M}$ is hyperelementary computable. 
As an application, we conclude from part (i) that the surgery obstruction groups $L_{n}(\mathbf{Z} G, \omega)$, with arbitrary orientation character $\omega: G \rightarrow\{ \pm 1\}$, are 2-hyperelementary computable after 2-adic completion (see Example 7.3 for the meaning of computability in this setting). This computability result was certainly expected to be true, but the argument presented here seems to be the first actual proof in the nonoriented case. In the oriented case, where $\omega$ is trivial, part (ii) applies to $L$-theory and the computability is just [Dress 1975, Theorem 1]. For nontrivial $\omega$, the surgery obstruction group $L_{n}(\mathbf{Z} G, \omega)$ is a Mackey functor if and only if it has exponent two (see [Taylor 1973], and see [Wall 1976, 5.2.5] for an example where the $L$-groups do not have exponent two). In Lemma 7.1 we give a general necessary and sufficient condition on $F$ for part (ii) to apply to $M$.

\section{Dress induction}

We will first recall some definitions Dress used in his formulation of induction theory [Dress 1975, page 301].

2A. Mackey functors. Let $G$ be a finite group, and let $\mathscr{D}(G)$ denote the category whose objects are finite, left $G$-sets and whose morphisms are $G$-maps. A Mackey functor is a bifunctor $\mathcal{M}=\left(\mathcal{M}_{*}, \mathcal{M}^{*}\right): \mathscr{D}(G) \rightarrow \mathscr{A} b$, where $\mathscr{A} b$ denotes the category of abelian groups and groups homomorphisms, such that $\mathcal{M}_{*}(S)=\mathcal{M}^{*}(S)$ for each object $S \in \mathscr{D}(G)$, and the following two properties hold:

(M1) For any pullback diagram of finite $G$-sets

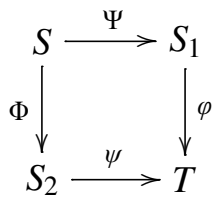

the induced maps give an commutative diagram

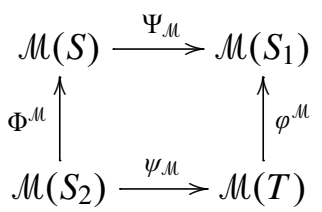

Here we denote the covariant maps by $\psi_{\mathcal{M}}$ and the contravariant maps by $\varphi^{\mu}$.

(M2) The embeddings of $S_{1}$ and $S_{2}$ into the disjoint union $S_{1} \sqcup S_{2}$ define an isomorphism $\mathcal{M}^{*}\left(S_{1} \sqcup S_{2}\right) \rightarrow \mathcal{M}^{*}\left(S_{1}\right) \oplus \mathcal{M}^{*}\left(S_{2}\right)$. Let $\mathcal{M}(\varnothing)=0$.

The property (M1) is the usual double coset formula, and (M2) gives additivity. 
We remark that for any bifunctor satisfying (M1), the composition

$$
\begin{aligned}
\mathcal{M}_{*}\left(S_{1}\right) \oplus \mathcal{M}_{*}\left(S_{2}\right) \rightarrow \mathcal{M}_{*}\left(S_{1} \sqcup S_{2}\right)= & \mathcal{M}^{*}\left(S_{1} \sqcup S_{2}\right) \\
& \rightarrow \mathcal{M}^{*}\left(S_{1}\right) \oplus \mathcal{M}^{*}\left(M_{2}\right)=\mathcal{M}_{*}\left(S_{1}\right) \oplus \mathcal{M}_{*}\left(S_{2}\right)
\end{aligned}
$$

is just the identity matrix. It follows that any subbifunctor of a Mackey functor is Mackey.

Definition 2.1. If $\mathcal{M}$ and $\mathcal{N}$ are Mackey functors, then a homomorphism $\mathcal{M} \rightarrow \mathcal{N}$ of Mackey functors is a natural transformation of bifunctors $\Theta: \mathcal{M} \rightarrow \mathcal{N}$ such that for each object $S \in \mathscr{D}(G)$ the function $\Theta_{S}: \mathcal{M}(S) \rightarrow \mathcal{N}(S)$ is a homomorphism of abelian groups. It is easy to check that the kernel, $\operatorname{ker} \Theta$, the image, $\operatorname{Im} \Theta$, and the cokernel of $\Theta$ are all sub- or quotient Mackey functors of $M$ or $\mathcal{N}$.

2B. Pairings and Green functors. If $\mathcal{M}, \mathcal{N}$, and $\mathscr{L}$ are Mackey functors, then a pairing is a family of bilinear maps

$$
\mu(S) \times \mathcal{N}(S) \rightarrow \mathscr{L}(S)
$$

indexed by the objects of $\mathscr{D}(G)$, such that for any $G$-map $\varphi: S \rightarrow T$ the following formulas hold:

$$
\begin{array}{ll}
\varphi^{\mathscr{L}}(x \cdot y)=\varphi^{\mathcal{M}}(x) \cdot \varphi^{\mathcal{N}}(y) & \text { for } x \in \mathcal{M}(T), y \in \mathcal{N}(T), \\
x \cdot \varphi_{\mathcal{N}}(y)=\varphi_{\mathscr{L}}\left(\varphi^{\mathcal{M}}(x) \cdot y\right) & \text { for } x \in \mathcal{M}(T), y \in \mathcal{N}(S), \\
\varphi_{\mathcal{M}}(x) \cdot y=\varphi_{\mathscr{L}}\left(x \cdot \varphi^{N}(y)\right) & \text { for } x \in \mathcal{M}(S), y \in \mathcal{N}(T) .
\end{array}
$$

A Green ring is a Mackey functor $\mathscr{G}$ together with a pairing $\mathscr{G} \times \mathscr{G} \rightarrow \mathscr{G}$, and a collection of elements $\left\{1_{S} \in \mathscr{G}(S)\right\}$ such that the pairing defines an associative ring structure on each $\mathscr{G}(S)$ with unit $1_{S}$, and $\varphi^{\mathscr{G}}\left(1_{T}\right)=1_{S}$ for every $G$-map $\varphi: S \rightarrow T$.

A homomorphism of Green rings $\Theta: \mathscr{G} \rightarrow \mathscr{K}$ is a homomorphism of Mackey functors such that for each object $S \in \mathscr{D}(G)$ the function $\Theta_{S}: \mathscr{G}(S) \rightarrow \mathscr{K}(S)$ is a unital ring homomorphism. If $\Theta_{S}$ is injective for each object $S \in \mathscr{D}(G)$, we say that $\varphi$ is a $s u b$-Green ring of $\mathscr{K}$. If $\Theta_{S}$ is surjective for each object $S \in \mathscr{D}(G)$, then we say that $\mathscr{K}$ is a quotient Green ring of $\mathscr{G}$. Similarly, we define subquotient Green rings.

If $\mathcal{M}$ is a Mackey functor, then $\mathcal{M}$ is a Green module over a Green ring $\varphi$ if there exists a pairing $\mathscr{G} \times \mathcal{M} \rightarrow \mathcal{M}$ such that $\mathcal{M}(S)$ becomes a left $\mathscr{G}(S)$-module from the pairing, and $1_{S} \cdot x=x$ for all $x \in \mathcal{M}(S)$.

Example 2.2. If $\mathscr{G} \rightarrow \mathscr{K}$ is a homomorphism of Green rings, then $\mathscr{K}$ is a Green module over $\mathscr{G}$ under the pairing $\mathscr{G} \times \mathscr{K} \rightarrow \mathscr{K}$ induced by the homomorphism. 
2C. The Burnside ring. For any left $G$-set $S$, we let $\mathscr{D}_{S}(G)$ denote the category with objects $(X, f)$, where $X$ is a left $G$-set and $f: X \rightarrow S$ is a $G$-map. The morphisms $F:\left(X_{1}, f_{1}\right) \rightarrow\left(X_{2}, f_{2}\right)$ are $G$-maps $F: X_{1} \rightarrow X_{2}$ such that $f_{2} \circ F=f_{1}$. We define a bifunctor

$$
\mathscr{A}: \mathscr{D}(G) \rightarrow \mathscr{A} b
$$

by setting $\mathscr{A}(S)=K_{0}\left(\mathscr{D}_{S}(G)\right)$. If $\varphi: S \rightarrow T$ is a $G$-map, then $\varphi_{\mathscr{A}}: \mathscr{A}(S) \rightarrow \mathscr{A}(T)$ is the map induced on $K_{0}$ by the composition $(X, f) \mapsto(X, \varphi \circ f)$. The contravariant map $\varphi^{\mathscr{A}}: \mathscr{A}(T) \rightarrow \mathscr{A}(S)$ is induced by the pullback construction applied to

$$
S \stackrel{\varphi}{\rightarrow} T \stackrel{f}{\leftarrow} Y,
$$

where $(Y, f)$ is an object in $\mathscr{D}_{T}(G)$. Conditions (M1) and (M2) are easy to check, and $\mathscr{A}$ is a Mackey functor. There is also a pairing $\mathscr{A} \times \mathscr{A} \rightarrow \mathscr{A}$ defined by pullback: let $\left(X_{1}, f_{1}\right)$ and $\left(X_{2}, f_{2}\right)$ represent elements of $\mathscr{A}(S)$, and form the pullback

$$
X_{1} \stackrel{f_{1}}{\longrightarrow} S \stackrel{f_{2}}{\longleftarrow} X_{2}
$$

considered as a $G$-set over $S$. This object in $\mathscr{D}_{S}(G)$ represents the product, and each $\mathscr{A}(S)$ becomes an associative ring with unit element represented by id: $S \rightarrow S$. The resulting Green ring is called the Burnside ring. Dress also remarks that the Burnside ring is the "universal" Green ring, since it acts on any Mackey functor $\mathcal{M}$. The required pairing $\mathscr{A} \times \mathcal{M} \rightarrow \mathcal{M}$ is defined by pairing an element of $\mathscr{A}(S)$ represented by a $G$-set $(X, f)$ over $S$, and an element $x \in \mathcal{M}(S)$, to get $f_{\mathcal{M}}\left(f^{\mathcal{M}}(x)\right) \in$ $\mathcal{M}(S)$. It is not hard to check that $\mathcal{M}(S)$ is a unital $\mathscr{A}(S)$-module under this bilinear pairing, so $\mathcal{M}$ is a Green module over $\mathscr{A}$.

We remark that a homomorphism $\mathcal{M} \rightarrow \mathcal{N}$ of Mackey functors is compatible with the $\mathscr{A}$-module action, so gives a map of $\mathscr{A}$-Green modules.

If $\mathscr{G}$ is a Green ring, the same checks show that $\mathscr{G}$ is an $\mathscr{A}$-algebra, implying in particular that $a \cdot(x \cdot y)=(a \cdot x) \cdot y$ for all $a \in \mathscr{A}(S)$ and all $x, y \in \mathscr{G}(S)$. It follows that the map $\imath: \mathscr{A} \rightarrow \mathscr{G}$ defined by $a \mapsto a \cdot 1_{S}$, for all $a \in \mathscr{A}(S)$, is a (unital) ring homomorphism. Indeed

$$
\left(a \cdot 1_{S}\right) \cdot\left(b \cdot 1_{S}\right)=a \cdot\left(1_{S} \cdot\left(b \cdot 1_{S}\right)\right)=a \cdot\left(b \cdot 1_{S}\right)=(a \cdot b) \cdot 1_{S}
$$

for all $a, b \in \mathscr{A}(S)$, since $\mathscr{G}(S)$ is a $\mathscr{A}(S)$-algebra. It is easy to check from the pairing formulas that $l: \mathscr{A} \rightarrow \mathscr{G}$ is also a homomorphism of Green rings.

2D. Ideals and quotient Green rings. There is a natural notion of a (left) Green ideal in a Green ring $\mathscr{G}$, namely a subbifunctor $I \subset \mathscr{G}$ such that $I(S) \subset \mathscr{G}(S)$ is a left ideal in the ring $\mathscr{G}(S)$. Similarly, we have right ideals and two-sided ideals. If $I \subset \mathscr{G}$ is a two-sided Green ideal, then the quotient functor $\mathscr{G} / I$, defined by $S \mapsto \mathscr{G}(S) / I(S)$, is a Green ring under the quotient pairing inherited from $\mathscr{G}$. 
If $\mathscr{G} \times \mathcal{M} \rightarrow \mathcal{M}$ is a Green module structure on a Mackey functor $\mathcal{M}$, then we define the Green ideal $I_{\mathcal{M}} \subset \mathscr{G}_{\text {as }}$ the subbifunctor of $\mathscr{G}_{\text {with }}$

$$
I_{\mathcal{M}}(S)=\left\{a \in \mathscr{G}(S) \mid \varphi \varphi(a) \cdot y=0, \psi^{\mathscr{G}}(a) \cdot z=0\right\}
$$

for all $\varphi: S \rightarrow T, \psi: U \rightarrow S$, and all $y \in \mathcal{M}(T), z \in \mathcal{M}(U)$. Note that elements of $I_{\mathcal{M}}(S)$ satisfy additional conditions (both "up" and "down") beyond just acting trivially on $M(S)$.

The pairing formulas show directly that $I_{\mathcal{M}}(S)$ is a two-sided ideal in the ring $\mathscr{G}(S)$, for every finite $G$-set $S$. We will check that $I_{\mathcal{M}}$ is a subbifunctor of $\mathscr{G}$ by looking at the operations induced by $G$-maps $\mu: V \rightarrow S$ and $\lambda: S \rightarrow W$ on an arbitrary element $a \in I_{\mathcal{M}}(S)$.

First we consider $\lambda \mathscr{G}(a) \in \mathscr{G}(W)$. Let $\varphi: W \rightarrow T$ and $\psi: U \rightarrow W$ be any $G$-maps. We have

$$
\left.\varphi_{\varphi}\left(\lambda_{\varphi}(a)\right) \cdot y=(\varphi \circ \lambda)\right)_{G}(a) \cdot y=0
$$

by definition of $I_{\mathcal{M}}(S)$. Let

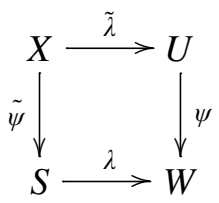

be the pullback square, and from (M1) we get

$$
\psi^{\mathscr{G}}(\lambda \varphi g(a)) \cdot z=\tilde{\lambda}_{g}\left(\tilde{\psi}^{\mathscr{G}}(a)\right) \cdot z=\tilde{\lambda}_{g}\left(\tilde{\psi}^{\mathscr{G}}(a) \cdot \tilde{\lambda}^{\mu}(z)\right)=0
$$

so $\lambda g(a) \in I_{M}(W)$.

Similarly, we must check that $\mu^{\varphi}(a) \in I_{M}(V)$. Let $\varphi: V \rightarrow T$ and $\psi: U \rightarrow V$ be $G$-maps, and note that

$$
\varphi \varphi\left(\mu^{\mathscr{G}}(a)\right) \cdot y=\varphi_{M}\left(\mu^{\mathscr{G}}(a) \cdot \varphi^{\mathcal{M}}(y)\right)=0
$$

and $\psi^{\varphi}\left(\mu^{\mathscr{G}}(a)\right) \cdot z=(\mu \circ \psi)^{\mathscr{G}}(a) \cdot z=0$.

We have now checked that $I_{\mathcal{M}} \subset \mathscr{G}$ is a subbifunctor, and therefore $I_{\mathcal{M}}$ is a Mackey functor and a two-sided Green ideal in $\varphi$. We define the quotient Green ring $\mathscr{G}_{\mathcal{M}}=\mathscr{G}_{/} / I_{\mathcal{M}}$ to be the bifunctor whose value on objects is given by the quotient

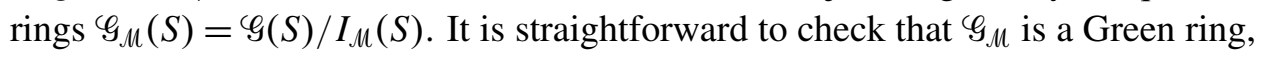
since the formulas above show that the pairing $\mathscr{G} \times \mathscr{G} \rightarrow \mathscr{G}$ restricts to pairings $I_{\mathcal{M}} \times \mathscr{G}_{\rightarrow} \rightarrow I_{\mathcal{M}}$ and $\mathscr{G} \times I_{\mathcal{M}} \rightarrow I_{\mathcal{M}}$ of Mackey functors. By construction, $\mathcal{M}$ is also a Green module over $\mathscr{G}_{\mu}$.

Definition 2.3. Let $\mathcal{M}$ be a Mackey functor. The Burnside quotient Green ring of $\mu$ is the Green ring $\mathscr{A}_{\mu}:=\mathscr{A} / I_{\mu}$. Let $\iota_{\mu}: \mathscr{A} \rightarrow \mathscr{A}_{\mu}$ denote the epimorphism of Green rings given by the natural quotient map. 
Remark 2.4. For $\mathscr{G}$ a Green ring, the map $\imath: \mathscr{A} \rightarrow \mathscr{G}$ defined above by $a \mapsto a \cdot 1_{S}$ factors through $\imath_{g}: \mathscr{A} \rightarrow A_{\mathscr{G}}$, and we obtain a canonical induced homomorphism

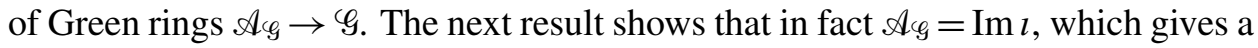
quick alternate definition of A\&. (For this observation, compare [Dress 1973, page 207; Oliver 1988, page 253; Hambleton and Taylor 2000, page 711; Bak 1995, page 236].)

Lemma 2.5. Let $G$ be a Green ring. Then the canonical homomorphism of Green rings, $A_{G} \rightarrow C_{G}$ is injective.

Proof. For each $G$-set $S$, the natural transformation of bifunctors in the statement maps $\mathscr{A}_{\mathscr{G}}(S) \rightarrow \mathscr{G}(S)$ by the ring homomorphism $a \mapsto a \cdot 1_{S}$, where $a \in \mathscr{A}(S)$ and $1_{S} \in \mathscr{G}(S)$ is the unit. If $a \cdot 1_{S}=0$, and $\varphi: S \rightarrow T, \psi: U \rightarrow S$ are $G$ maps, then it follows as above that $\varphi_{\mathscr{A}}(a) \cdot 1_{T}=0$ and $\psi^{\mathscr{A}}(a) \cdot 1_{U}=0$, Therefore $\left\{a \in \mathscr{A}(S) \mid a \cdot 1_{S}=0\right\} \subset I_{\mathscr{G}}(S)$, and the ring homomorphism $\mathscr{A}_{\mathscr{G}}(S) \rightarrow \mathscr{G}(S)$ is injective.

We will explore Definition 2.3 by considering the Burnside quotient Green rings for filtrations of Mackey functors.

Definition 2.6. If $\mathcal{M}$ and $\mathcal{N}$ are Mackey functors, we say that $\mathcal{M}$ is a subfunctor of $\mathcal{N}$ (respectively $\mathcal{N}$ is a quotient functor of $\mathcal{M}$ ) if there is a natural transformation $\Theta: \mathcal{M} \rightarrow \mathcal{N}$ such that for each object $S \in \mathscr{D}(G)$ the function $\Theta_{S}: \mathcal{M}(S) \rightarrow \mathcal{N}(S)$ is an injective (respectively, surjective) homomorphism of abelian groups. We say that $\mathcal{M}$ is a subquotient of $\mathcal{N}$ if there is a finite sequence of Mackey functors $\mathcal{M}=\mathscr{L}_{0}, \mathscr{L}_{1}, \ldots, \mathscr{L}_{r}=\mathcal{N}$ such that each $\mathscr{L}_{i}$ is either a subfunctor or a quotient functor of $\mathscr{L}_{i+1}$, for $i=0, \ldots, r-1$. Note that the relation " $M$ is a subquotient of $\mathcal{N}$ " is a transitive relation.

Example 2.7. If $\Theta: \mathscr{G} \rightarrow \mathscr{K}$ is a homomorphism of Green rings, then we can regard $\mathscr{K}$ as a Green module over $\mathscr{G}$. Furthermore, $\operatorname{ker} \Theta=I_{\mathscr{K}} \subset \mathscr{G}$, and there is an induced homomorphism $\mathscr{G}_{\mathscr{K}} \rightarrow \mathscr{K}$ of Green rings. If $\mathscr{K}$ is a quotient Green ring of $\mathscr{G}$, then $\mathscr{K}=\mathscr{G}_{\mathscr{K}}=\mathscr{G} / I_{\mathscr{K}}$.

Lemma 2.8. Let $\mathscr{G}$ be a Green ring and $M$ a Green module over $G$. Then the Burnside quotient Green ring $\mathscr{A}_{M}$ is a quotient of $\mathscr{A}_{\mathrm{g}}$, and isomorphic to a subquotient of $\varphi$.

Proof. Since $A_{G}$ is a sub-Green ring of $\mathscr{G}$, we just need to check that $\mathscr{A}_{\mu}$ is a quotient Green ring of $A_{\mathscr{G}}$ under the natural projection from $\mathscr{A}$. This is equivalent to the statement that $I_{\varphi} \subset I_{\mu}$. Let $a \in I_{G}(S)$, and consider $G$-maps $\varphi: S \rightarrow T$ and $\psi: U \rightarrow S$. For any $y \in \mathcal{M}(T)$,

$$
\varphi_{\mathscr{A}}(a) \cdot y=\varphi_{\mathscr{A}}(a) \cdot\left(1_{T} \cdot y\right)=\left(\varphi_{\mathscr{A}}(a) \cdot 1_{T}\right) \cdot y=0,
$$


since $1_{T} \in \mathscr{G}(T)$. Similarly, for any $z \in \mathcal{M}(U)$,

$$
\psi^{\mathscr{A}}(a) \cdot z=\psi^{\mathscr{A}}(a) \cdot 1_{U} \cdot z=0,
$$

and we see that $a \in I_{\mathcal{M}}(S)$.

Lemma 2.9. Let $\mathcal{M}$ and $\mathcal{N}$ be Mackey functors, with $\mathcal{M}$ a subquotient of $\mathcal{N}$. Then there is a surjective homomorphism of Green rings $\mathfrak{f}: \mathscr{A}_{\mathcal{N}} \rightarrow \mathscr{A}_{\mathcal{M}}$ such that $\mathfrak{f} \circ l_{\mathcal{N}}=$ $\imath_{\mu}$.

Proof. We will establish this result for subfunctors and quotient functors, and note that the general subquotient case follows by an inductive argument on the length of the chain joining $M$ to $\mathcal{N}$.

Suppose first that $\Theta: M \rightarrow \mathcal{N}$ is a natural transformation, with $\Theta_{S}: \mathcal{M}(S) \rightarrow \mathcal{N}(S)$ injective for all finite $G$-sets $S$. Let $a \in I_{\mathcal{N}}(S)$ and let $\varphi: S \rightarrow T, \psi: U \rightarrow S$ be $G$-maps. Then for any $y \in M(T), \Theta_{T}\left(\varphi_{\mathscr{A}}(a) \cdot y\right)=\varphi_{\mathscr{A}}(a) \cdot\left(\Theta_{T}(y)\right)=0$ since $\Theta$ is a $\mathscr{A}$-Green module map. Similarly, for any $z \in \mathcal{M}(U), \Theta_{U}\left(\psi^{\mathscr{A}}(a) \cdot z\right)=$ $\psi^{\mathscr{A}}(a) \cdot\left(\Theta_{U}(z)\right)=0$. Since $\Theta_{T}$ and $\Theta_{U}$ are injective, it follows that $a \in I_{M}(S)$, and $I_{\mathcal{N}} \subset I_{\mathcal{M}}$ so that $\mathscr{A}_{\mathcal{N}}$ maps onto $\mathscr{A}_{\mathcal{M}}$.

Next suppose that $\Theta: \mathcal{N} \rightarrow \mathcal{M}$ is a natural transformation, with $\Theta_{S}: \mathcal{N}(S) \rightarrow \mathcal{M}(S)$ surjective for all finite $G$-sets $S$. If $a \in I_{\mathcal{N}}(S)$, we check that $\varphi_{\mathscr{A}}(a) \cdot y=0$ and $\psi^{\mathscr{A}}(a) \cdot z=0$, for all $y \in \mathcal{M}(T)$ and all $z \in \mathcal{M}(U)$, by using the surjectivity of $\Theta_{T}$ and $\Theta_{U}$, and the compatibility of $\Theta$ with the $\mathscr{A}$-module structures on $\mathcal{M}$ and $\mathcal{N}$. Therefore $I_{\mathcal{N}} \subset I_{\mathcal{M}}$.

In general, if $\mathcal{M}$ is a sub-Mackey functor of $\mathcal{N}$ it is not true that $I_{\mathcal{M}} \subset I_{\mathcal{N}}$, so there is no natural map in the other direction from $\mathscr{A}_{\mu}$ onto $\mathscr{A}_{\mathcal{N}}$, but here is one more situation that works.

We say that $\mathcal{M}$ is a full lattice in $\mathcal{N}$ if there is a natural transformation $\Theta: M \rightarrow$ $\mathcal{N}$ such that the induced maps $\Theta_{S}^{*}: \operatorname{Hom}(\mathcal{N}(S), \mathcal{N}(S)) \rightarrow \operatorname{Hom}(\mathcal{M}(S), \mathcal{N}(S))$ are injective for all finite $G$-sets $S$. Note that $\mathcal{M}$ need not be a sub-Mackey functor of $\mathcal{N}$ for this condition to hold.

Lemma 2.10. Let $\mathcal{M}$ and $\mathcal{N}$ be Mackey functors, with $M$ be a full lattice in $\mathcal{N}$. Then there exists a surjective homomorphism of Green rings $\mathfrak{g}: \mathscr{A}_{\mathcal{M}} \rightarrow \mathscr{A}_{\mathcal{N}}$ such that $\mathfrak{g} \circ \imath_{M}=\imath_{N}$. If $\mathcal{M}$ is also a subfunctor of $\mathcal{N}$, then $\mathfrak{g}$ is an isomorphism and the inverse to the $\mathfrak{f}: \mathscr{A}_{\mathcal{N}} \rightarrow \mathscr{A}_{\mathcal{M}}$ described previously.

Proof. Let $\varphi: S \rightarrow T$ be a $G$-map. For each $a \in \mathscr{A}(S)$ we can consider the action map $y \mapsto \varphi_{\mathscr{A}}(a) \cdot y$ as an element of $\operatorname{Hom}(\mathcal{N}(T), \mathcal{N}(T))$. However if $a \in I_{\mathcal{M}}(a)$, this homomorphism is zero on the image of $\Theta_{T}$, and therefore it vanishes identically. Similarly, we check that $\psi^{\mathscr{A}}(a) \cdot z=0$ for all $z \in \mathcal{N}(U)$ and any $G$-map $\psi: U \rightarrow S$. Therefore $I_{\mathcal{M}} \subset I_{\mathcal{N}}$. 
2E. Amitsur complexes. Dress proves computation results for Mackey functors via the contractibility of certain chain complexes. Let $X, Y$ be finite $G$-sets, and define a semisimplicial set $\operatorname{Am}(X, Y)$ inductively. Let $\operatorname{Am}_{0}(X, Y)=Y$ and $A m_{r}(X, Y)=X \times A m_{r-1}(X, Y)$ for $r \geq 1$. There are $G$-maps

$$
d_{i}^{r}: A m_{r}(X, Y) \rightarrow A m_{r-1}(X, Y)
$$

for $0 \leq i<r$, defined by setting $d_{0}^{r}$ as the projection

$$
X \times A m_{r-1}(X, Y) \rightarrow A m_{r-1}(X, Y),
$$

and for $i>0$ by $d_{i}^{r}=1_{X} \times d_{i-1}^{r-1}$.

Definition 2.11. Let $\mathcal{M}$ be a Mackey functor. For given finite $G$-sets $X, Y$, the Amitsur complex $\mathcal{M}(A m(X, Y))$ is the chain bicomplex whose chain group in dimension $r$ is $\mathcal{M}\left(A m_{r}(X, Y)\right)$, with boundary operators $\partial_{r}=\sum(-1)^{i}\left[d_{i}^{r}\right]_{\mathcal{M}}$ and $\delta^{r}=\sum(-1)^{i}\left[d_{i}^{r}\right]^{\mu}$ for $r \geq 0$. and zero otherwise.

This construction has certain naturality properties.

Lemma 2.12. Let $M$ be a Mackey functor. The Amitsur complex gives a bifunctor

$$
M\left(A m\left({ }_{-},-\right)\right): \mathscr{D}(G) \times \mathscr{D}(G) \rightarrow \operatorname{Chain}(\mathscr{A} b)
$$

where Chain $(A b)$ denotes the category of chain complexes of abelian groups.

For any Mackey functor $\mathcal{M}$, and any finite $G$-set $S$, let $\mathcal{M}_{S}$ denote the Mackey functor defined by $\mathcal{M}_{S}(T)=\mathcal{M}(S \times T)$, for any finite $G$-set $T$. There are natural transformations and $\Theta_{S}^{\mu}: \mathcal{M} \rightarrow \mathcal{M}_{S}$ and $\Theta_{\mathcal{M}}^{S}: \mathcal{M}_{S} \rightarrow \mathcal{M}$ of Mackey functors induced by the projection maps $S \times T \rightarrow T$. Dress says that $\mathcal{M}$ is $S$-injective (respectively $S$-projective) if $\Theta_{S}^{\mathcal{M}}$ is split-injective (respectively $\Theta_{\mathcal{M}}^{S}$ is split surjective).

Lemma 2.13 [Dress 1975, Proposition 1.1']. A Mackey functor $M$ is S-injective if and only if it is $S$-projective.

Proof. Suppose that $\mathcal{M}$ is $S$-projective, so that $\Theta_{\mathcal{M}}^{S}$ is split-injective. Let $\Phi: M \rightarrow M_{S}$ be a natural transformation such that $\Theta_{\mathcal{M}}^{S} \circ \Phi=I d_{\mathcal{M}}$ (the identity natural transformation on $M$ ). If $\Delta: S \rightarrow S \times S$ denotes the diagonal map and $p: S \times T \rightarrow T$ the second factor projection, we notice that

$$
S \times T \stackrel{\Delta \times 1}{\longrightarrow} S \times S \times T \stackrel{1 \times p}{\longrightarrow} S \times T
$$

is just the identity map on $S \times T$. It follows that

$$
\Theta_{\mathcal{M}(T)}^{S} \circ(\Delta \times 1)^{\mathcal{M}} \circ \Phi_{S \times T} \circ \Theta_{S}^{\mathcal{M}(T)}=I d_{\mathcal{M}(T)}
$$

for any finite $G$-set $T$. One can check that the formula

$$
\widetilde{\Phi}(T):=\Theta_{\mathcal{M}(T)}^{S} \circ(\Delta \times 1)^{\mathcal{M}} \circ \Phi_{S \times T}
$$


defines a natural transformation of bifunctors splitting $\Theta_{S}^{\mu}$ and hence $\mathcal{M}$ is $S$ injective. The converse is similar.

Dress now proves that, for any finite $G$-set $Y$ and whenever $M$ is $S$-injective or $S$-projective, both Amitsur complexes $\left(\mu_{*}(A m(S, Y), \partial)\right.$ and $\left(\mu^{*}(A m(S, Y), \delta)\right.$ are contractible (we say $\mathcal{M}$ is $S$-computable). In particular, for $Y=\bullet$ there are exact sequences

$$
\begin{aligned}
& \cdots \stackrel{\partial_{3}}{\longrightarrow} M(S \times S) \stackrel{\partial_{2}}{\longrightarrow} M(S) \stackrel{\partial_{1}}{\longrightarrow} M(\bullet) \longrightarrow 0 \\
& 0 \longrightarrow M(\bullet) \stackrel{\delta_{1}}{\longrightarrow} M(S) \stackrel{\delta_{2}}{\longrightarrow} M(S \times S) \stackrel{\delta_{3}}{\longrightarrow} \cdots
\end{aligned}
$$

which exhibit $M(\bullet)$ as a limit of induction or restriction maps respectively.

Here is the main theorem of Dress induction theory:

Proposition 2.14 [Dress 1975, Proposition 1.2]. Let $\mathscr{G}$ be a Green ring and $S$ be a finite $G$-set. Then the following conditions are equivalent:

(1) The map $\varphi g: \mathscr{G}(S) \rightarrow \mathscr{G}(\bullet)$ associated to the projection $\varphi: S \rightarrow \bullet$ is surjective.

(2) $\mathscr{G}$ is $S$-injective.

(3) All $\varphi$-modules are $S$-injective.

This result focuses attention on the task of finding a suitable Green ring which acts on $\mathcal{M}$, and then checking property (i). We remark that the Burnside ring $\mathscr{A}$ acts on any Mackey functor, but $\mathscr{A}$ is $S$-injective only if $\bullet \subset S$. Hence the Burnside ring itself has no useful induction properties.

\section{Dress generating sets}

In the classical Mackey setting of $G$-functors given by Green [1971], computation is expressed in terms of families. A family of subgroups $\mathscr{F}$ of $G$ is a collection of subgroups closed under conjugation and taking subgroups. For any finite $G$-set $X$ let $\mathscr{F}(X)$ denote the family generated by the isotropy subgroups of $X$. For example, the family $\mathscr{F}(\bullet)=\{$ All $\}$. Conversely, given a family $\mathscr{F}$ of subgroups, we can form the disjoint union $X(\mathscr{F})$ of $G$-sets $G / H$, one for each conjugacy class of maximal elements in $\mathscr{F}$, under the partial ordering from subgroup inclusion. For example, $X(\{\mathrm{All}\})=\bullet$. We say that a family of subgroups $\mathscr{F}$ contracts a Mackey functor $\mathcal{M}$ if and only if $\mathcal{M}$ is $X(\mathscr{F})$-projective or $X(\mathscr{F})$-injective.

We have seen that a good strategy for computing a Mackey functor $M$ is to study the Green rings acting on $M$. We will apply this strategy to the Burnside quotient Green ring $\mathscr{A}_{\mathcal{M}}$ of $\mathcal{M}$.

Definition 3.1. Let $\mathscr{G}$ be a Green ring. A finite $G$-set $X$ is a generating set for $\mathscr{G}$ if the natural map $\mathscr{G}(X) \rightarrow \mathscr{G}(\bullet)$ is surjective (equivalently, if $1 . \in \operatorname{Im}\{\mathscr{G}(X) \rightarrow \mathscr{G}(\bullet)\})$. 
By [Dress 1975, Proposition 1.2], $X$ is a generating set for $\varphi$ if and only if $\varphi$ is $X$ injective or $X$-projective. It is not true in general that a generating set for a Green ring $\mathscr{G}$ is also a generating set for the sub-Green ring $A_{\mathscr{C}}$. To obtain generation for $A_{\varphi g}$ it is usually necessary to enlarge the generating set.

For $H$ a finite group and $p$ a prime, let

$$
O^{p}(H)=\bigcap\left\{H_{0} \triangleleft H \mid H / H_{0} \text { is a } p \text {-group }\right\}
$$

Notice that $O^{p}(H)$ is a characteristic subgroup of $p$-power index in $H$, and

$$
O^{p}\left(\left(O^{p}(H)\right)=O^{p}(H) .\right.
$$

Definition 3.2. Let $\mathscr{F}$ be a family of subgroups of $G$ and $p$ a prime. Then hyper $-\mathscr{F}$ is the family consisting of all subgroups $H$ in $G$ such that $O^{p}(H) \in \mathscr{F}$. If $S$ is a $G$-set, then hyper $_{p}-S$ is the corresponding $G$-set to hyper $_{p}-\mathscr{F}(S)$. This construction is due to Dress [1975, page 307].

It is easy to check that hyper - $^{-}$is closed under taking subgroups and conjugation, so we obtain a family of subgroups. By construction, there is a $G$-map $X \rightarrow$ hyper $_{p}-X$ for any $X$ and hyper $_{p}$-hyper $_{p}-X=$ hyper $_{p}-X$. One of Dress's main results is the following:

Theorem 3.3 [Dress 1973, page 207]. Let M be a Mackey functor. For any prime $p$ and for any finite $G$-set $Y$, let $\mathscr{K}(Y)=\operatorname{ker}\left(\mathcal{M}(\bullet) \otimes \mathbf{Z}_{(p)} \rightarrow \mathcal{M}(Y) \otimes \mathbf{Z}_{(p)}\right)$ and $\Phi(Y)=\operatorname{Im}\left(M\left(\right.\right.$ hyper $\left.\left._{p}-Y\right) \otimes \mathbf{Z}_{(p)} \rightarrow \mathcal{M}(\bullet) \otimes \mathbf{Z}_{(p)}\right)$. Then $\mathcal{M}(\bullet) \otimes \mathbf{Z}_{(p)}=\mathscr{K}(Y)+\mathscr{I}(Y)$.

If $Y$ is a finite $G$-set, we will use the notation $\langle Y\rangle$ for the equivalence class of $Y$ in the category $\mathscr{D}(G)$. One useful consequence is:

Lemma 3.4. Let $\mathscr{G}_{0}$ be a sub-Green ring of $\mathscr{G}_{1}$. For any prime $p$, and any finite $G$-set $Y$ with $\langle Y\rangle=\left\langle\right.$ hyper $\left._{p}-Y\right\rangle$, the natural map $\mathscr{G}_{0}(Y) \otimes \mathbf{Z}_{(p)} \rightarrow \mathscr{G}_{0}(\bullet) \otimes \mathbf{Z}_{(p)}$ is surjective if and only if $\varphi_{1}(Y) \otimes \mathbf{Z}_{(p)} \rightarrow \mathscr{G}_{1}(\bullet) \otimes \mathbf{Z}_{(p)}$ is surjective.

Proof. For any Green ring $\mathscr{G}$ and any finite $G$-set $Y$, the image of $\mathscr{G}(Y) \otimes \mathbf{Z}_{(p)}$ in $\mathscr{G}(\bullet) \otimes \mathbf{Z}_{(p)}$ is an ideal. Hence either map is onto if and only if $1 \varphi_{i}(\bullet)$ is in the image. Since $1 \varphi_{0}(\bullet)$ goes to $1 \varphi_{1}(\bullet)$, this proves the first implication.

For the converse, the surjectivity of $\varphi_{1}(Y) \otimes \mathbf{Z}_{(p)} \rightarrow \mathscr{G}_{1}(\bullet) \otimes \mathbf{Z}_{(p)}$ implies that the Amitsur complex is contractible for the restriction maps induced by the transformation $Y \rightarrow \bullet$. In particular, $\mathscr{G}_{1}(\bullet) \otimes \mathbf{Z}_{(p)} \rightarrow \mathscr{G}_{1}(Y) \otimes \mathbf{Z}_{(p)}$ is injective. Therefore $\mathscr{G}_{0}(\bullet) \otimes \mathbf{Z}_{(p)} \rightarrow \mathscr{G}_{0}(Y) \otimes \mathbf{Z}_{(p)}$ is injective, and from Theorem 3.3 we conclude that $\mathscr{G}_{0}\left(\right.$ hyper $\left._{p}-Y\right) \otimes \mathbf{Z}_{(p)} \rightarrow \mathscr{G}_{0}(\bullet) \otimes \mathbf{Z}_{(p)}$ is surjective.

Suppose that $G$ is a Green ring which acts on a Mackey functor $\mathcal{M}$. For many applications of induction theory, the "best" Green ring for $\mathcal{M}$ is the Burnside quotient Green ring $A_{g}$. This is a Green ring which acts on $\mathcal{M}$, and by construction

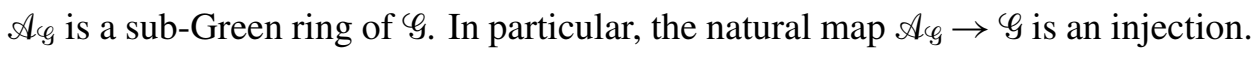


Definition 3.5. A finite $G$-set $X$ is a Dress generating set for a Green ring $\mathscr{G}$, provided that $\mathscr{G}\left(\right.$ hyper $\left._{p}-X\right) \otimes \mathbf{Z}_{(p)} \rightarrow \mathscr{G}(\bullet) \otimes \mathbf{Z}_{(p)}$ is surjective for each prime $p$.

By Theorem 3.3, any finite $G$-set $X$ such that the natural map $\mathscr{G}(\bullet) \rightarrow \mathscr{G}(X)$ is injective is a Dress generating set for $\mathscr{G}$. Notice that a Dress generating set for $\mathscr{G}$ is also a Dress generating set for any quotient Green ring of $\mathscr{G}$. The following result (Theorem A) is the main step in handling sub-Green rings.

Theorem 3.6. A finite $G$-set $X$ is a Dress generating set for a Green ring $\mathcal{G}$ if and only if it is a Dress generating set for the Burnside quotient Green ring Acg.

Proof. We apply the result above to $Y=$ hyper $_{p}-X$, for each prime $p$, and note that A $g$ is a sub-Green ring of $\mathscr{G}$.

The Burnside quotient Green ring can be used to compute Mackey functors obtained by subquotients.

Definition 3.7. A finite $G$-set $X$ is a Dress generating set for a Mackey functor $\mathcal{M}$, provided that $X$ is a Dress generating set for the Burnside quotient Green ring $\mathscr{A}_{\mu}$ of $\mathcal{M}$.

This is consistent with our previous Definition 3.5 for a Green ring.

Theorem 3.8. Let $\mathscr{G}$ be a Green ring and $\mathcal{M}, \mathcal{N}$ Mackey functors.

(i) If $\mathcal{M}$ is a $\varphi_{\text {-module and } X}$ is a Dress generating set for $\mathscr{G}$, then $X$ is a Dress generating set for $M$.

(ii) If $\mathcal{N}$ is a subquotient of $\mathcal{M}$ and $X$ is a Dress generating set for $\mathcal{M}$, then $X$ is a Dress generating set for $\mathcal{N}$.

(iii) If $\mathcal{M}$ is a full lattice in $\mathcal{N}$ and $X$ is a Dress generating set for $\mathcal{M}$, then $X$ is a Dress generating set for $\mathcal{N}$.

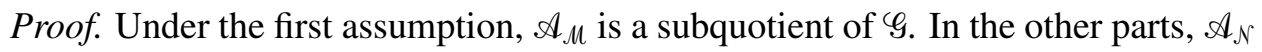
is a quotient of $\mathscr{A}_{\mu}$.

We can translate this into a computability statement as follows:

Corollary 3.9. Let $p$ be a prime and $\varphi$ be a Green ring. Suppose that F is a hyper ${ }_{p}$-closed family of subgroups of $G$. Then $\mathscr{G} \otimes \mathbf{Z}_{(p)}$ is $\mathscr{F}$-computable if and only if $A_{\varphi} \otimes \mathbf{Z}_{(p)}$ is $\mathscr{F}$-computable.

The advantage of $A_{\varphi}$ over $\mathscr{G}$ is that $A_{\varphi}$ acts on Mackey functors which are subfunctors or quotient functors of $\mathcal{M}$ but $\mathscr{G}$ does not in general. For example, $\mathscr{G}$ never acts on $A_{\varphi}$ unless they are equal. We next point out another good feature of the Burnside quotient Green ring. 
Theorem 3.10 [Hambleton 2006, Theorem 1.8]. Suppose that $\varphi$ is a Green ring which acts on a Mackey functor $\mathcal{M}$, and $\mathscr{F}$ is a hyper ${ }_{p}$-closed family of subgroups

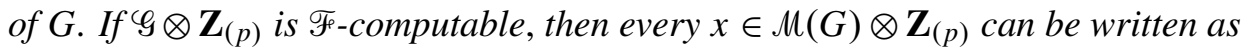

$$
x=\sum_{H \in \mathscr{F}} a_{H} \operatorname{Ind}_{H}^{G}\left(\operatorname{Res}_{G}^{H}(x)\right)
$$

for some coefficients $a_{H} \in \mathbf{Z}_{(p)}$, where the $a_{H}$ are the same for all $x$.

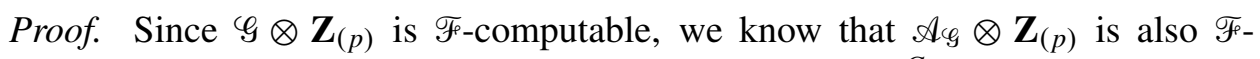
computable. Therefore, we can write $1=\sum_{K \in \mathscr{F}} b_{K} \operatorname{Ind}_{K}^{G}\left(y_{K}\right)$ for some $y_{K} \in$ $\mathscr{A}_{\mathscr{G}}(K) \otimes \mathbf{Z}_{(p)}$ and $b_{K} \in \mathbf{Z}_{(p)}$. For any $x \in \mathcal{M}(G) \otimes \mathbf{Z}_{(p)}$ we now have the formula

$$
x=1 \cdot x=\sum_{K \in \mathscr{F}} b_{K} \operatorname{Ind}_{K}^{G}\left(y_{K} \cdot \operatorname{Res}_{G}^{K}(x)\right) .
$$

But each $y_{K} \in \mathscr{A}_{\varphi}(K) \otimes \mathbf{Z}_{(p)}$ can be represented by a sum $\sum c_{K H}[K / H]$, with $c_{K H} \in \mathbf{Z}_{(p)}$, under the surjection $\mathscr{A}(K) \rightarrow A_{\varphi}(K)$. Therefore

$$
\begin{aligned}
x & =\sum_{K \in \mathscr{F}} b_{K} \sum_{H \subseteq K} c_{K H} \operatorname{Ind}_{K}^{G}\left([K / H] \cdot \operatorname{Res}_{G}^{K}(x)\right) \\
& =\sum_{K \in \mathscr{F}} b_{K} \sum_{H \subseteq K} c_{K H} \operatorname{Ind}_{K}^{G}\left(\operatorname{Ind}_{H}^{K}\left(\operatorname{Res}_{K}^{H}\left(\operatorname{Res}_{G}^{K}(x)\right)\right)\right) \\
& =\sum_{K \in \mathscr{F}} b_{K} \sum_{H \subseteq K} c_{K H} \operatorname{Ind}_{H}^{G}\left(\operatorname{Res}_{G}^{H}(x)\right)
\end{aligned}
$$

We now define $a_{H}=\sum_{K \in \mathscr{F}} b_{K} c_{K H}$, and the formula becomes

$$
x=\sum_{H \in \mathscr{F}} a_{H} \operatorname{Ind}_{H}^{G}\left(\operatorname{Res}_{G}^{H}(x)\right) .
$$

Example 3.11 (Representation theory). Recall that a $p$-(hyper)elementary group is a (semi)direct product $C \rtimes P$, where $P$ is a $p$-group and $C$ is cyclic of order prime to $p$. A Dress generating set for a Green ring $\mathscr{G}$ need not be a generating set for $\mathscr{G}$. For example, let $E$ denote the finite $G$-set, $E=\bigsqcup G / H$, where we have one $H$ for each $p$-elementary subgroup of $G$. It is known that $E$ is a generating set for the complex representation ring $R_{\mathbf{C}}(G)$, but not in general for the rational representation ring $R_{\mathbf{Q}}(G)$. On the other hand, complex representations are detected by characters, so any $G$-set with isotropy containing the cyclic family is a Dress generating set for $R_{\mathbf{C}}(G)$, or for the sub-Green ring $R_{\mathbf{Q}}(G)$ by Theorem 3.8 (ii). It follows that the hyperelementary family $\mathscr{H}$ gives a generating set $X_{\mathscr{H}}$ for $R_{\mathbf{Q}}(G)$. This implies the Brauer-Berman-Witt induction theorem for rational representations.

Example 3.12 (The Swan ring). The Swan ring is one of the main examples of Green rings in the classical setting of induction theory [Swan 1970]. For any finite group, let $S W(G, \mathbf{Z})$ denote the Grothendieck group of isomorphism classes of 
finitely-generated left $\mathbf{Z} G$-modules, with $[L]=\left[L^{\prime}\right]+\left[L^{\prime \prime}\right]$ whenever there is a short exact sequence

$$
0 \rightarrow L^{\prime} \rightarrow L \rightarrow L^{\prime \prime} \rightarrow 0
$$

of such $\mathbf{Z} G$-modules. The operation $L \otimes_{\mathbf{Z}} L^{\prime}$ gives a ring structure on this Grothendieck group, so we obtain a commutative ring. The usual induction and restriction operations for such modules give the Swan ring the structure of a Mackey functor. We let

$$
S W_{G}: \mathscr{D}(G) \rightarrow \mathscr{A} b
$$

denote the Green ring (in the sense of Dress) defined by $S W_{G}(G / H):=S W(H, \mathbf{Z})$, and extended to $\mathscr{D}(G)$ by additivity. Since $S W(G, \mathbf{Z})$ is hyperelementary computable by Swan's induction theorem [Dress 1973, page 211], we see that any Mackey functor on which this Green ring acts is hyperelementary computable.

It follows that the Burnside quotient Green ring of the Swan ring, denoted $\mathscr{A}_{S W}$, also has the hyperelementary set $X_{\mathscr{H}}$ as a Dress generating set (or more precisely, any $G$-set whose isotropy contains the cyclic family is a Dress generating set). In this case, $\mathscr{A}_{S W}(G / H) \subset S W_{G}(G / H)$ is the subring $P(H, \mathbf{Z}) \subset S W(H, \mathbf{Z})$ generated by the permutation modules $\mathbf{Z}[H / K]$, for all subgroups $K \subseteq H$.

\section{Computation techniques}

Dress generating sets can also be used to compute exact sequences of Mackey functors or filtrations of Mackey functors by subfunctors. We say that

$$
M_{0} \stackrel{a}{\rightarrow} M_{1} \stackrel{b}{\rightarrow} M_{2}
$$

is an exact sequence of Mackey functors if $a$ and $b$ are homomorphisms of Mackey functors, such that the sequence $\mu_{0}(S) \rightarrow \mu_{1}(S) \rightarrow \mu_{2}(S)$ is exact for each finite $G$-set $S$. We define long exact sequences in a similar way.

Proposition 4.1. Suppose that $\mu_{0} \rightarrow M_{1} \rightarrow M_{2}$ is an exact sequence of Mackey functors. Then $X$ is a Dress generating set for $M_{1}$ whenever $X$ is a Dress generating set for $M_{0}$ and $M_{2}$.

Proof. We may assume that $\mu_{0} \rightarrow M_{1}$ is injective, and that $\mu_{1} \rightarrow M_{2}$ is surjective, and the projections from $\mathscr{A}$ induce a natural transformation $\theta: \mathscr{A}_{M_{1}} \rightarrow \mathscr{A}_{M_{0}} \oplus \mathscr{A}_{M_{2}}$ of Green rings. By exactness, $I_{\theta}(S):=\operatorname{ker} \theta_{S}$ is a nilpotent ideal (of nilpotence index 2). Let $\bar{A}_{M_{1}}=\mathscr{A}_{M_{1}} / I_{\theta}$ denote the quotient Mackey functor. Since this Mackey functor is mapped injectively by $\theta$ into $\mathscr{A}_{\mathcal{M}_{0}} \oplus \mathscr{A}_{\mathcal{M}_{2}}, X$ is a Dress generating set for $\bar{A}_{M_{1}}$. It follows that $\overline{\mathscr{A}}_{M_{1}}\left(\right.$ hyper $\left._{p}-X\right) \otimes \mathbf{Z}_{(p)} \rightarrow \overline{\mathscr{A}}_{M_{1}}(\bullet) \otimes \mathbf{Z}_{(p)}$ is surjective for every prime $p$. But an element in $\mathscr{A}_{\mathcal{M}_{1}}(\bullet) \otimes \mathbf{Z}_{(p)}$ hitting $1 . \in \bar{A}(\bullet) \otimes \mathbf{Z}_{(p)}$ has the form $1+u$, where $u \in I_{\theta}(\bullet) \otimes \mathbf{Z}_{(p)}$. Since $u^{2}=0,1+u$ is invertible and $(1+u)^{-1}=1-u$. If $p_{X}: X \rightarrow \bullet$ denotes the projection map, and $\left(p_{X}\right)_{*}(a)=1+u$, then we have 
$\left(p_{X}\right)_{*}\left(\left(p_{X}\right)^{*}(1 \bullet-u) \cdot a\right)=(1 \bullet-u) \cdot\left(p_{X}\right)_{*}(a)=1$. and hence $X$ is a Dress generating set for $\mathscr{A}_{\mathcal{M}_{1}}$.

Remark 4.2. In the proof of Proposition 4.1, we have shown for each prime $p$, there exists an element $a \in \mathscr{A}\left(\right.$ hyper $\left._{p}-X\right) \otimes \mathbf{Z}_{(p)}$ such that $a \mapsto 1$. in each of the Burnside quotient Green rings $\mathscr{A}_{\mathcal{M}_{i}}(\bullet) \otimes \mathbf{Z}_{(p)}$, for $i=0,1,2$. The same argument extends by induction to finite filtrations of a Mackey functor by sub-Mackey functors.

Corollary 4.3. Let $0=\mathcal{N}_{0} \subset \cdots \subset \mathcal{N}_{r}=\mathcal{M}$ be a filtration of a Mackey functor by sub-Mackey functors. Then $X$ is a Dress generating set for $\mathcal{M}$ if and only if $X$ is a Dress generating set for each quotient $\mathcal{N}_{i} / \mathcal{N}_{i-1}$, for $1 \leq i \leq r$.

A finite length chain complex of Mackey functors is a sequence $\left(\mathcal{N}_{i}, \partial_{i}\right)$ of Mackey functors $\mathcal{N}_{i}, 0 \leq i \leq r$, and natural transformations $\partial_{i}: \mathcal{N}_{i} \rightarrow \mathcal{N}_{i-1}, 1 \leq i \leq r$, such that $\left(\mathcal{N}(S), \partial_{*}\right)$ is a chain complexes of abelian groups for each finite $G$-set $S$. A chain complex $\mathcal{N}$ of Mackey functors has homology groups $H_{i}(\mathcal{N}), 0 \leq i \leq r$, which are subquotient Mackey functors of $\mathcal{N}_{i}$.

Corollary 4.4. Suppose that $\mathcal{N}$ is a finite length chain complex of Mackey functors. If $X$ is a Dress generating set for each $\mathfrak{N}_{i}, 0 \leq i \leq r$, then $X$ is a Dress generating set for each of the homology Mackey functors $H_{i}(\mathcal{N}), 0 \leq i \leq r$.

Another useful construction is completion.

Theorem 4.5. Let $M$ be a Mackey functor, and let $\mathscr{F}$ denote a (possibly infinite) filtration

$$
M=F_{0} \supseteq F_{1} \supseteq \cdots \supseteq F_{r} \supseteq \cdots
$$

of $M$ by sub-Mackey functors. A finite $G$-set $X$ is a Dress generating set for $\widehat{M}_{\mathscr{F}}=\lim _{\leftarrow} M / F_{r}$ if and only if $X$ is a Dress generating set for each quotient Mackey functor $\overleftarrow{F}_{r-1} / F_{r}, r \geq 1$.

Proof. Since each $F_{r-1} / F_{r}$ is a subquotient of $\widehat{M}_{\mathscr{F}}$, the necessity follows from the results above. For sufficiency, we first note by Corollary 4.3 that $X$ is Dress generating set for each quotient $M / F_{r}$. It is enough to prove that $X$ generates the inverse limit $\lim \mathscr{A}_{\mathcal{M} / F_{r}}$ of the Burnside quotient Green rings for the sequence $\left\{\mathcal{M} / F_{r}\right\}$. Suppose that $X$ is a Dress generating set for each $\mathscr{A}_{\mathcal{M} / F_{r}}, r \geq 1$, and set $Y=$ hyper $_{p}-X$. If $\left\{a_{r}\right\}$ is a sequence of elements in $\mathscr{A}_{\mathcal{M} / F_{r}}(Y)$ hitting $1_{\bullet}^{r}$, we can use the contractibility of the $Y$-Amitsur complex for $\mathscr{A}_{\mathcal{M} / F_{r+1}}$ inductively, to adjust each $a_{r+1}$ by an element of $\mathscr{A}_{\mathcal{M} / F_{r+1}}(Y \times Y)$, so that $a_{r+1} \mapsto a_{r}$. This gives us an element in the inverse limit $\lim _{\longleftarrow} \mathbb{A}_{\mathcal{M} F_{r}}(Y)$ hitting $1 . \in \lim \mathscr{A}_{\mathcal{M} F_{r}}(\bullet):=\mathscr{G}(\bullet)$, and hence the Green ring $\mathscr{G}$ acts on $\widehat{M}_{\mathscr{F}}$ with $X$ as a Dress generating set. Since $A_{G} \rightarrow \mathscr{A}_{\widehat{\mathcal{M}}_{\mathscr{F}}}$ is surjective, it follows that $\widehat{M}_{\mathscr{F}}$ has $X$ as a Dress generating set. 
Example 4.6. Here is an important special case. Let $\mathscr{G}$ be a Green ring acting on a Mackey functor $\mathcal{M}$. If $\mathscr{I} \subset \mathscr{G}$ is a Green ideal, we may filter $\mathcal{M}$ by the subbifunctors $F_{r}=\Phi^{r} \mathcal{M}$ and then $\widehat{M}_{\mathscr{F}}$ is the $\mathscr{I}$-adic completion of $\mathcal{M}$.

In particular, for a given Mackey functor $\mathcal{M}$ we could take $\mathscr{I}=\left\langle\mathscr{I}_{\mathcal{M}}, 2\right\rangle$, and then $\widehat{M}_{\mathscr{I}}$ is just the 2-adic completion of the Mackey functor $\mathcal{M}$. Note that if $\mathcal{M}(X)$ is finitely generated, then $\widehat{M}_{\Phi}(X) \cong \mathcal{M}(X) \otimes \widehat{\mathbf{Z}}_{2}$.

\section{Mackey functors and $R G$-Morita}

To prove Theorem B we need to define the bifunctor $d: \mathscr{D}(G) \rightarrow R G$-Morita used in its statement. This involves some definitions and elementary properties of categories with bisets as morphisms, which are well-known to the experts. We include this material for the reader's convenience.

In [HTW 1990, 1.A.4] we introduced the category $R G$-Morita whose basic objects are finite groups $H$ isomorphic to some subquotient of $G$, and whose morphisms were defined by a Grothendieck group construction on the isomorphism classes of finite $H_{2}-H_{1}$ bisets $X$, for which the order of the left stabilizer

$$
H_{2} I(x)=\left\{h \in H_{2} \mid h x=x\right\}
$$

is a unit in $R$, for all $x \in X$. Here $R$ is a commutative ring with unit. We set $X \sim X^{\prime}$ if $R X$ is isomorphic to $R X^{\prime}$ as $R H_{2}-R H_{1}$ bimodules. The balanced product $X \times_{H_{2}} Y$ of an $H_{3}-H_{2}$ biset $X$ and an $H_{2}-H_{1}$ biset $Y$ is a $H_{3}-H_{1}$ biset. This defines the composition for morphisms. The Add-construction [MacLane 1971, page 194] is then applied to complete the definition. Many functors arising in algebraic $K$ theory and topology are actually functors out of $R G$-Morita, so it is of interest to recognize when these are Mackey functors.

To relate Mackey functors and $R G$-Morita, we will need the $G$-Burnside category, $\mathbf{A}(G)$, whose objects are subgroups $H \subset G$, and where $\operatorname{Hom}_{\mathbf{A}(G)}\left(H_{1}, H_{2}\right)$ is the Grothendieck construction applied to the isomorphism classes of finite bifree $H_{2}-H_{1}$ bisets (meaning both left and right actions are free). Because of the Grothendieck group construction, $\mathbf{A}(G)$ is an Ab-category, the morphism sets are abelian groups and the compositions are bilinear [MacLane 1971, I.8, page 28]. Let

$$
u: \mathbf{A}(G) \rightarrow \mathbf{A} .(G)
$$

denote the associated universal free additive category, and the universal inclusion [MacLane 1971, VII.2, problem 6, page 194].

The morphisms in $\mathbf{A}(G)$ are defined by the Grothendieck group construction with addition operation the disjoint union of bisets. By convention, the empty biset $\varnothing$ represents the zero element. Composition comes from the balanced product:

$$
{ }_{H_{3}} X_{H_{2}} \circ{ }_{H_{2}} X_{H_{1}}=\left({ }_{H_{3}} X_{H_{2}}\right) \times{ }_{H_{2}}\left({ }_{H_{2}} X_{H_{1}}\right) .
$$


The reader should check that this is well-defined on isomorphism classes of bisets and "bilinear" in that

$$
\left({ }_{H_{3}} X_{H_{2}} \sqcup{ }_{H_{3}} Y_{H_{2}}\right){ }_{H_{2}} X_{H_{1}} \cong\left({ }_{H_{3}} X_{H_{2}} \circ{ }_{H_{2}} X_{H_{1}}\right) \sqcup\left({ }_{H_{3}} Y_{H_{2}} \circ{ }_{H_{2}} X_{H_{1}}\right),
$$

with a similar formula for disjoint union on the right. The morphisms in $\mathbf{A} .(G)$ are matrices of morphisms in $\mathbf{A}(G)$.

Definition 5.1. We define a contravariant involution $\tau: \mathbf{A}(G) \rightarrow \mathbf{A}(G)$, by the identity on objects, and on morphisms it is the map induced on the Grothendieck construction by the function which takes the finite bifree $H_{2}-H_{1}$ biset ${ }_{H_{2}} X_{H_{1}}$ to the finite bifree $H_{1}-H_{2}$ biset ${ }_{H_{1}} X_{H_{2}}$ which is $X$ as a set and $h_{1} \cdot x \cdot h_{2}$ is defined to be $h_{2}^{-1} x h_{1}^{-1}$.

The reader needs to check that isomorphic bisets are isomorphic after reversing the order, and should also check that the transpose conjugate of a disjoint union is isomorphic to the disjoint union of the conjugate transposes of the pieces. This means that $\tau$ is a functor which induces a homomorphism of Hom-sets. It is clearly an involution, not just up to natural equivalence. Since $\tau$ is a homomorphism on Hom-sets, it induces an additive contravariant involution $\tau^{\bullet}: \mathbf{A} \bullet(G) \rightarrow \mathbf{A} \bullet(G)$, called conjugate transpose, which commutes with the functor $u$. By definition, $\tau$ • acts on a matrix of morphisms by applying $\tau$ to each entry, and then transposing the matrix. There is a functor

$$
a: \mathbf{A .}(G) \rightarrow R G \text {-Morita }
$$

given by the inclusion on objects and morphisms (but the equivalence relation on morphisms is different in $R G$-Morita).

There is a functor $\mathbf{A} .(G) \rightarrow R$-Morita, called the $R$-group ring functor, where $R$-Morita has objects $R$-algebras and morphisms defined by stable isomorphism classes of bimodules [HTW 1990, 1.A.1]. This functor factors through $R G$-Morita: it sends $H \mapsto R H$ on objects, and $X \mapsto R X$ on morphisms.

We will define the following diagram of categories and functors:

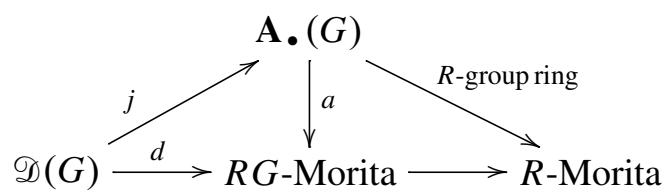

To complete the definition of the functors in this diagram, we need to introduce another category. Let $\mathscr{D}_{*}(G)$ denote the category whose objects are pairs $(X, \mathbf{b})$, consisting of a finite $G$-space $X$ and an ordered collection $\mathbf{b}=\left(b_{1}, \ldots, b_{n}\right)$ of basepoints, one for each $G$-orbit of $X$. The morphisms are the $G$-maps (not necessarily 
base-point preserving). There is a functor

$$
\mu: \mathscr{D}_{*}(G) \rightarrow \mathscr{D}(G)
$$

defined by forgetting the base-points. Since every object of $\mathscr{D}(G)$ is isomorphic to the image $\mu(X, \mathbf{b})$ of an object of $\mathscr{D}_{*}(G)$, and $\mu$ induces a bijection on morphism sets, it follows that $\mu$ gives an equivalence between the categories $\mathscr{D}_{*}(G)$ and $\mathscr{D}(G)$, with inverse functor $\mu^{\prime}$ [MacLane 1971, IV.4, Theorem 1, page 91].

We can now define two functors

$$
\left(j \bullet, j^{\bullet}\right): \mathscr{D}_{*}(G) \rightarrow \mathbf{A} \bullet(G) .
$$

The covariant functor $j \bullet$ is the additive extension of the functor which sends an object $(G / H, e H)$ to the isotropy subgroup $H$, and sends the $G$ map $f: G / H \rightarrow G / K$ to the biset ${ }_{K} K_{g^{-1} H g}$, where $f(e H)=g K$. If we change the coset representative and write $f(e H)=g_{1} K$, then the map

$$
\psi:{ }_{K} K_{g^{-1} H g} \rightarrow{ }_{K} K_{g_{1}^{-1} H g_{1}}
$$

defined by $\psi(k)=k\left(g^{-1} g_{1}\right)$ gives an bijection of $K$ - $K$ bisets.

Note that $1_{G / H}: G / H \rightarrow G / H$ goes to ${ }_{H} H_{H}$, which is the identity. Check that if $f_{1}: G / H_{1} \rightarrow G / H_{2}$ and $f_{2}: G / H_{2} \rightarrow G / H_{3}$ are $G$-maps and if $f_{1}\left(e H_{1}\right)=g_{1} H_{2}$ and $f_{2}\left(e H_{2}\right)=g_{2} H_{3}$ then $f_{2} \circ f_{1}\left(e H_{1}\right)=\left(g_{1} g_{2}\right) H_{3}$ and

$$
H_{3}\left(H_{3}\right)_{g_{2}^{-1} H_{2} g_{2}} \times{ }_{H_{2} H_{2}}\left(H_{2}\right)_{g_{1}^{-1} H_{1} g_{1}}
$$

is isomorphic to ${ }_{H_{3}}\left(H_{3}\right)_{\left(g_{1} g_{2}\right)^{-1} H_{1}\left(g_{1} g_{2}\right)}$ by the map $\left(h_{3}, h_{2}\right) \mapsto h_{3} g_{2}^{-1} h_{2} g_{2}$.

The contravariant functor $j \bullet$ agrees with $j$. on objects, but sends the $G$ map $f: G / H \rightarrow G / K$ to the biset ${ }_{g^{-1}} \mathrm{Hg}_{\mathrm{K}} K_{\mathrm{K}}$, where $f(e H)=g K$. Rather than checking the identity and composition directly, just note that ${ }_{g^{-1}} \mathrm{Hg}_{\mathrm{K}} K_{K}$ is isomorphic to $\tau\left({ }_{K} K_{g^{-1} H g}\right)$ by the function which sends $k$ to $k^{-1}$, so $j^{\bullet}=\tau \circ j$. and hence $j^{\bullet}$ is a contravariant functor.

Definition 5.4. We define the bifunctor

$$
j: \mathscr{D}(G) \rightarrow \mathbf{A} \cdot(G)
$$

as the composition $j=\left(j^{\bullet}, j_{\bullet}\right) \circ \mu^{\prime}$. Let

$$
d=a \circ j: \mathscr{D}(G) \rightarrow R G \text {-Morita }
$$

denote the composition in diagram (5.2).

For any additive functor $F: \mathbf{A} .(G) \rightarrow \mathscr{A} b$, the composition $F \circ j: \mathscr{D}(G) \rightarrow \mathscr{A} b$ is a Mackey functor [HTW 2008]. Our main application is the following: 
Theorem 5.5. Any additive functor $F: R G$-Morita $\rightarrow$ Ab gives a Mackey functor on $\mathscr{D}(G)$ by composition with $d: \mathscr{D}(G) \rightarrow R G$-Morita. Any such Mackey functor is hyperelementary computable.

Proof. The functor $d: \mathscr{D}(G) \rightarrow R G$-Morita factors through $\mathbf{A}_{\bullet}(G)$, so we obtain Mackey functors by composition. We will show that any such Mackey functor, $M$, is a Green module over the Burnside quotient Green ring $\mathscr{A}_{S W}$ of the Swan ring, and then apply Example 3.12. Let $L=\mathbf{Z}[H / K]$ denote a permutation module, for some subgroups $K \subset H$ of $G$, and let $X$ denote an $H$ - $H$-biset, which is free as a left $H$-set. Then $H / K \times X$ is again an $H$ - $H$-biset by the formula $h_{1}(h K, x) h_{2}=$ $\left(h_{1} h K, h_{1} x h_{2}\right)$, for all $h, h_{1}, h_{2} \in H$ and all $x \in X$. Note that $R[H / K \times X]=$ $L \otimes_{\mathbf{Z}} R X$ as $R H-R H$ bimodules, so this construction applied to $X={ }_{H} H_{H}$, sending $\mathbf{Z}[H / K] \mapsto H / K \times H$, gives a well-defined homomorphism

$$
P(H, \mathbf{Z}) \rightarrow \operatorname{Hom}_{R G \text {-Morita }}(H, H)
$$

from the Grothendieck group of permutation modules, for each subgroup $H \subset G$. The adjoints of these homomorphisms give a pairing $\mathscr{A}_{S W} \times \mathcal{M} \rightarrow \mathcal{M}$, and the Green module properties follow easily from bimodule identities (compare [Oliver 1988, 11.2]). Since $\mathscr{A}_{S W}$ is hyperelementary computable, we conclude that any Mackey functor out of $R$-Morita is hyperelementary computable.

Remark 5.6. As mentioned in the Introduction, this is a refinement of an earlier result of Oliver [1988, 11.2]. Oliver establishes hyperelementary computability for functors of the form $X(R[G])$, where $X$ is an additive functor from the category of $R$-orders in semisimple $K$-algebras with bimodule morphisms to the category of abelian groups. Here $R$ is a Dedekind domain with quotient field $K$ of characteristic zero.

There are two points of comparison: it should first be noted that Oliver [1988, page 246] is dealing with Mackey functors defined on the category of finite groups and monomorphisms, so the statement that any such functor $X(R[G])$ is a Mackey functor is straight-forward. In our case, relating $R G$-Morita to Mackey functors defined on finite $G$-sets in the sense of Dress [1975, page 301] involves some work (for example, in constructing the bifunctor $d$ ). The translation between the two versions of Mackey functors is also well-known to the experts [Dress 1975, Section 1], but in this paper we preferred to work only with the Dress $G$-set theory.

The second point of comparison is that Oliver's proof uses an action of the Swan ring on the Mackey functors $X(R[G])$, but the Swan ring does not act on our functors in any obvious way. The key new ingredient in our proof is the Burnside quotient Green ring of the Swan ring. Apart from this additional input, the argument is essentially the same. However, the extra generality can be useful since 
there are functors out of $R G$-Morita which do not appear to extend to the setting of Oliver [1988, 11.2].

Example 5.7 (Controlled topology). The bounded categories $\mathscr{C}_{M, G}(R)$ of [Hambleton and Pedersen 1991, §4], and the continuously controlled categories $\mathscr{B}_{G}(X \times$ $[0,1) ; R)$ of [Hambleton and Pedersen 2004, §6] are functors out of $\mathbf{A}$. $(G)$, for any finite group $G$, and hence any additive functor from these categories to abelian groups gives a Mackey functor on $\mathscr{D}(G)$.

Example 5.8 (Farrell-Hsiang induction). There is a useful extension of induction theory to (possibly) infinite groups, due to Farrell and Hsiang [1977]. Given any representation $\mathrm{pr}: \Gamma \rightarrow G$, with $G$ finite, we get a new $R$-group ring functor $\mathscr{A}(G) \rightarrow R$-Morita by sending $G / H \mapsto R\left[\Gamma_{H}\right]$, where $\Gamma_{H}=\operatorname{pr}^{-1}(H)$ is the preimage of $H$ in $\Gamma$. We have a generating set for the morphisms $\operatorname{Hom}_{\mathbf{A}(G)}\left(H_{1}, H_{2}\right)$ consisting of the bisets $H_{2} \times_{K} H_{1}$, where $K \subset H_{2} \times H_{1}$ is a subgroup [HTW 1990, 1.A.9]. We send the biset $H_{2} \times_{K} H_{1}$ to the bimodule $R\left[\Gamma_{H_{2}}\right] \otimes_{R\left[\Gamma_{K}\right]} R\left[\Gamma_{H_{1}}\right]$. By composition with any additive functor $F: R$-Morita $\rightarrow \mathscr{A} b$, we again obtain Mackey functors. Since the Swan ring acts on $R$-Morita (by tensor product as above), any such Mackey functor is a Green module over the Swan ring, and we obtain hyperelementary computation as before. The main examples are listed in [HTW 1990, 1.A.12], including Quillen $K$-theory $K_{n}(R[\Gamma])$.

Remark 5.9. An alternate (and slightly sharper) formulation of this example could be given by defining $R \Gamma$-Morita for any discrete group $\Gamma$ : the objects are finite groups $H$ isomorphic to some subquotient $H \cong \Gamma_{1} / \Gamma_{0}$ of $\Gamma$, where $\Gamma_{0} \triangleleft \Gamma_{1}$ and $\Gamma_{1}$ is finite index in $\Gamma$. The morphisms are $H_{2}-H_{1}$ bisets as before. Then from any representation pr: $\Gamma \rightarrow G$, where $G$ is finite, we get a functor $d: \mathscr{D}(G) \rightarrow$ $R \Gamma$-Morita and Theorem 5.5 holds in this new setting.

Example 5.10 (Cohomotopy). Example 2.1 and Remark 2.4 (pages 108-109) of [Lam 1968] show that (ordinary or Tate) cohomology with twisted coefficients $H^{i}($ ?; $M)$ is a Mackey functor on $\mathscr{D}(G)$ where $M$ is a fixed $G$-module. Since the cohomotopy Green ring $H \mapsto \pi^{0}(B H)$ acts on this Mackey functor, it is Sylow computable. If $\mathrm{pr}: \Gamma \rightarrow G$ is a homomorphism and $M$ is a $\Gamma$-module, then $H^{i}\left(\operatorname{pr}^{-1}(?) ; M\right)$ is also a Mackey functor on $\mathscr{D}(G)$ with the cohomotopy Green ring acting. An interesting example of this situation is Galois cohomology.

\section{Pseudo-Mackey functors and pseudo-complexes}

We wish to apply the computation strategy described above to a more general situation, namely to study functors which have induction and restriction but are not known to be Mackey. The main examples of interest are the higher Whitehead groups $\mathrm{Wh}_{n}(\mathbf{Z} G)$ and the nonoriented surgery obstruction groups $L_{n}(\mathbf{Z} G, \omega)$. 
Definition 6.1. A covariant prefunctor $f: \mathscr{D} \rightarrow \mathscr{E}$ between two categories is just a function $S \mapsto o b(f)(S)$ on objects, and a function

$$
\operatorname{hom}(f): \operatorname{Hom}_{\mathscr{D}}\left(S_{1}, S_{2}\right) \rightarrow \operatorname{Hom}_{\mathscr{E}}\left(o b(f)\left(S_{1}\right), o b(f)\left(S_{2}\right)\right)
$$

on Hom-sets. A functor is a prefunctor which preserves identities and compositions. Similarly, we define a contravariant prefunctor, and a prebifunctor then consists of a pair $\left(f_{*}, f^{*}\right)$ of prefunctors, where $f_{*}$ is covariant, $f^{*}$ is contravariant, and $o b\left(f_{*}\right)=o b\left(f^{*}\right)$. We call these Mackey prefunctors if $\mathscr{D}=\mathscr{D}(G)$ and $\mathscr{E}=\mathscr{A} b$.

A prenatural transformation $T: f_{1} \rightarrow f_{2}$ is a function

$$
S \mapsto T(S) \in \operatorname{Hom}_{\mathscr{E}}\left(o b\left(f_{1}\right)(S), o b\left(f_{2}\right)(S)\right) .
$$

A natural transformation of (covariant) prefunctors is a prenatural transformation $T: f_{1} \rightarrow f_{2}$ such that the diagram

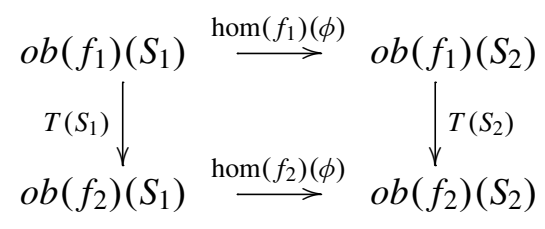

commutes for all pairs of objects $S_{1}, S_{2} \in \mathscr{D}$ and all $\phi \in \operatorname{Hom}_{\mathscr{D}}\left(S_{1}, S_{2}\right)$. There is a similar definition for (pre)natural transformations of contravariant prefunctors, and a natural transformation of prebifunctors is a single function which is natural transformation for both the covariant and contravariant parts of the bifunctor. A prepairing between three Mackey prefunctors $\mathcal{M}, \mathcal{N}$ and $\mathscr{L}$ is a collection of functions $\mu(S): \mathcal{M}(S) \times \mathcal{N}(S) \rightarrow \mathscr{L}(S)$. Finally, if $\mathcal{M} \rightarrow \mathcal{N}$ is an injective natural transformation of Mackey prefunctors, then we say that $\mathcal{M}$ is a sub-Mackey prefunctor of $\mathcal{N}$.

Note that if $\mathcal{M} \mathscr{D}(G) \rightarrow \mathscr{A} b$ is a Mackey prefunctor, we can apply $\mathcal{M}$ to any of the Amitsur complexes $\operatorname{Am}(X, Y)$, and obtain $\partial_{r}$ and $\delta^{r}$ maps as usual, but we can not be sure that $\partial_{r} \circ \partial_{r+1}=0$ or $\delta^{r+1} \circ \delta^{r}=0$. We call $\mathcal{M}(A m(X, Y))$ a pre-Amitsur complex. This construction gives a prefunctor $\mathscr{D}(G) \times \mathscr{D}(G) \rightarrow$ Chain $(\mathscr{A} b)$.

Definition 6.2. A Mackey prefunctor $\mathcal{M}$ is called a pseudo-Mackey functor provided that there exists a finite collection of Mackey prefunctors $0=\mathcal{N}_{0} \subset \mathcal{N}_{1} \subset$ $\cdots \subset \mathcal{N}_{r}=\mathcal{M}$ such that the quotient prebifunctors $\mathcal{N}_{i} / \mathcal{N}_{i-1}$ are actually Mackey functors, for $1 \leq i \leq r$. The collection $\left\{\mathcal{N}_{i} / \mathcal{N}_{i-1} \mid 1 \leq i \leq r\right\}$ will be called the associated graded Mackey functor to $\mathcal{M}$.

A natural transformation $\mathcal{M} \rightarrow \mathcal{N}$ of pseudo-Mackey functors is a natural transformation of Mackey prefunctors which preserves the filtrations. Notice that the Burnside ring $\mathscr{A}$ acts on a Mackey prefunctor via the usual formula (which gives a 
prepairing). The action of $\mathscr{A}$ on a pseudo-Mackey functor $\mathcal{M}$ preserves the filtration, and the induced action on the subquotients $\mathcal{N}_{i} / \mathcal{N}_{i-1}$ is the usual action.

We say that a finite $G$-set $X$ is a Dress generating set for a pseudo-Mackey functor $\mathcal{M}$ provided $X$ is a Dress generating set for each of the Mackey functors $\mathcal{N}_{i} / \mathcal{N}_{i-1}$ in its associated filtration. This agrees with our previous definitions if $\mu$ is a Mackey functor filtered by Mackey subfunctors. Notice that the image of the natural map of Green rings $\mathscr{A} \rightarrow \bigoplus_{i=1}^{r} \mathscr{A}_{\mathcal{N}_{i} / \mathcal{N}_{i-1}}$ is a Green ring with $X$ as a Dress generating set. It follows that there exists an element $a \in \mathscr{A}\left(\right.$ hyper $\left._{p}-X\right) \otimes \mathbf{Z}_{(p)}$, for each prime $p$, whose image in $\mathscr{A}(\bullet) \otimes \mathbf{Z}_{(p)}$ acts as 1 . on each subquotient $\mathcal{N}_{i} / \mathcal{N}_{i-1}(\bullet) \otimes \mathbf{Z}_{(p)}, 1 \leq i \leq r$.

Lemma 6.3. Suppose that $\mu_{0} \rightarrow \mu_{1}$ and $\mu_{1} \rightarrow M_{2}$ are natural transformations of Mackey prefunctors, such that $\mu_{0}(Y) \rightarrow \mu_{1}(Y) \rightarrow M_{2}(Y)$ is exact for every finite $G$-set $Y$. If $M_{0}$ and $M_{2}$ are pseudo-Mackey functors, then $M_{1}$ is a pseudo-Mackey functor. Moreover, if $X$ is a Dress generating set for $M_{0}$ and $M_{2}$, then $X$ is a Dress generating set for $M_{1}$.

Proof. The preimage of the associated filtration for $\mu_{2}$ gives a filtration $\mathcal{N}_{0} \subset$ $\mathcal{N}_{1} \subset \cdots \subset \mathcal{N}_{r}=M_{1}$, with $\mathcal{M}_{0} \subset \mathcal{N}_{i}$ for $0 \leq i \leq r$. Since a subbifunctor of a Mackey functor is Mackey, we see that the quotient prefunctors $\mathcal{N}_{i} / \mathcal{N}_{i-1}$ are actually Mackey functors (and they all have Dress generating set $X$ by Theorem 3.8). Now we extend this filtration by adjoining the associated filtration for $M_{0}$. Since each of the subquotients in this extended filtration have Dress generating set $X$, the result follows.

We also get a computational result for pseudo-Mackey functors. The Amitsur precomplex $\left(M_{*}\left(A m(X, Y), \partial_{*}\right)\right.$ is now a pseudo-complex, meaning that the boundary maps $\partial_{*}$ are filtration-preserving (and the associated graded is an actual complex). It will be called pseudo-contractible if it is equipped with degree +1 filtration-preserving natural transformations

$$
s_{r}: M\left(A m_{r}(X, Y)\right) \rightarrow M\left(A m_{r+1}(X, Y)\right)
$$

of prefunctors, for $r \geq 0$, which contract the Amitsur complexes for the associated graded Mackey functors to $\mathcal{M}$. The collection $s_{*}=\left\{s_{r}\right\}$ is called a pseudocontraction. We make a similar definition for the cochain Amitsur complex and the degree -1 cochain pseudo-contractions $\sigma^{r}$.

We can construct pseudo-contractions by using any element $a \in \mathscr{A}(X)$ such that $a$ acts as $1 . \in$ on each subquotient $\mathcal{N}_{i} / \mathcal{N}_{i-1}(\bullet) \otimes \mathbf{Z}_{(p)}, 1 \leq i \leq r$, to build chain homotopies $s_{r}(a)$ and cochain homotopies $\sigma^{r}(a)$. These are pseudo-contractions in the above sense. 
Proposition 6.4. Let $\mathcal{M}$ be a pseudo-Mackey functor, and $X, Y$ finite $G$-sets. If $\left(M_{*}\left(A m(X, Y), \partial_{*}\right)\right.$ is pseudo-contractible with pseudo-contraction $s_{*}$, then there are canonical filtration-preserving natural transformations $\left(\partial_{*}^{\prime}, s_{*}^{\prime}\right)$ for which $s_{*}^{\prime}$ is a chain contraction and $\left(\mathcal{M}_{*}\left(A m(X, Y), \partial_{*}^{\prime}\right)\right.$ is a chain complex. If the pseudocomplex was already a complex, $\partial_{*}^{\prime}=\partial_{*}$, and if in addition $s_{*}$ was already a contraction, then $s_{*}^{\prime}=s_{*}$.

Proof. Let $\left(C_{i}, \partial_{i}, s_{i}\right)$ be our data, where $\partial_{i}$ and $s_{i}$ are natural transformations. We assume that for $i<r, \partial_{i} \circ \partial_{i+1}=0, \partial_{i+1}=\partial_{i+1} \circ s_{i} \circ \partial_{i+1}$, and $s_{i-1} \circ \partial_{i}+\partial_{i+1} \circ s_{i}=1_{C_{i}}$. For $r \leq 0$ these identities clearly hold. We proceed to show how these conditions may be achieved for $i=r$ by modifying $\partial_{r+1}$ and $s_{r}$ (if necessary). Throughout the inductive construction, we do not change the maps induced by $\left(\partial_{*}, s_{*}\right)$ on the Amitsur complex for the associated graded Mackey functor to $\mathcal{M}$. We also note that the process does not change the given $\partial_{1}: C_{1} \rightarrow C_{0}$, but may change $s_{0}$ in the first step.

First, let $\partial_{r+1}^{\prime}=\partial_{r+1}-s_{r-1} \circ \partial_{r} \circ \partial_{r+1}$. Then $\partial_{r} \circ \partial_{r+1}^{\prime}=0$ and if $\partial_{r} \circ \partial_{r+1}=0$ we have $\partial_{r+1}^{\prime}=\partial_{r+1}$. Note that both $\partial_{r+1}^{\prime}$ and $\partial_{r+1}$ preserve the induced filtration from $\mathcal{M}$, and induce the same map on the Amitsur complexes for the associated graded Mackey functor to $M$.

Next, we modify $s_{r}$. Let $\psi_{r}=s_{r-1} \circ \partial_{r}+\partial_{r+1}^{\prime} \circ s_{r}$. By construction, $\psi_{r}$ preserves the filtration and induces the identity on the associated graded. Hence, $\psi_{r}=1_{C_{r}}+u$, where $u$ is nilpotent, and $\psi_{r}$ is invertible. Since $\partial_{r} \circ \psi_{r}=\partial_{r}$, we can set $s_{r}^{\prime}=s_{r} \circ \psi_{r}^{-1}$ and obtain $s_{r-1} \circ \partial_{r}+\partial_{r+1}^{\prime} \circ s_{r}^{\prime}=1_{C_{r}}$ by precomposing with $\psi_{r}$. Notice that if $s_{r}$ was already part of a chain contraction, then we do not alter it. It follows that $\partial_{r+1}^{\prime}=\partial_{r+1}^{\prime} \circ s_{r}^{\prime} \circ \partial_{r+1}^{\prime}$ and the induction step is complete. The naturality of $\partial_{r}^{\prime}$ and $\psi_{r}$ follow inductively from the explicit formulas. The naturality of $\psi_{r}$ implies the naturality of $s_{r}^{\prime}$ for use at the next step of the induction. Since no choices were involved in the construction of $\left(\partial_{*}^{\prime}, s_{*}^{\prime}\right)$, the new maps are canonically determined by the original data $\left(\partial_{*}, s_{*}\right)$.

Remark 6.5. After this process, the new contractible complex gives an expression for $\mathcal{M}(Y)$ as a direct summand of $\mathcal{M}(X \times Y)$, with respect to the original induction map $\partial_{1}: \mathcal{M}(X \times Y) \rightarrow \mathcal{M}(Y)$, and the new restriction map $s_{0}^{\prime}: \mathcal{M}(Y) \rightarrow: \mathcal{M}(X \times Y)$, since $\partial_{1} \circ s_{0}^{\prime}=\mathrm{id}$. In this situation, we say that $M(Y)$ is computed from the family $\mathscr{F}(X)$. If $\mathcal{M}$ was actually a Mackey functor, computability is this sense would agree with the notion previously defined. Similar remarks apply to the contravariant version $\mathcal{M}^{*}\left(A m(X, Y), \delta^{*}\right)$.

We will also need a slight extension of this result. A filtered precomplex $(C, \partial)$ is a precomplex of abelian groups equipped with a filtration

$$
C=F_{0} C \supset F_{1} C \supset F_{2} C \supset \cdots
$$


where each $F_{i} C$ is a presubcomplex of $(C, \partial)$, meaning that $\partial_{r}\left(F_{i} C_{r}\right) \subseteq F_{i} C_{r-1}$, for all $i, r$. We say that $(C, \partial)$ is a pseudo-complex if the additional relation $\partial_{r} \circ \partial_{r+1}=0$ holds, for all $r$, on each subquotient $F_{i} C / F_{i+1} C$. We say that a pseudo-complex has a pseudo-contraction $s_{*}=\left(s_{r}\right)$ provided that $s_{r}\left(F_{i} C_{r}\right) \subseteq F_{i} C_{r+1}$, and $s_{*}$ induces an actual contraction on each subquotient complex $F_{i} C / F_{i+1} C$.

A pseudo-complex $(C, \partial)$ has a natural completion

$$
(C, \partial) \longrightarrow \lim _{\leftarrow} C / F_{i} C:=(\widehat{C}, \widehat{\partial})
$$

given by the inverse limit precomplex with respect to the natural projections $C \rightarrow$ $C / F_{i} C, i \geq 0$. A pseudo-contraction $s_{*}$ of $(C, \partial)$ induces a precontraction $\hat{s}_{*}$ of $(\widehat{C}, \widehat{\partial})$.

Proposition 6.6. Let $(C, \partial)$ be a pseudo-complex with filtration $\left\{F_{i} C \mid i \geq 0\right\}$. If $(C, \partial)$ admits a filtered pseudo-contraction $s_{*}$, then there exists canonical data for which $\left(\widehat{C}, \partial_{*}^{\prime}, s_{*}^{\prime}\right)$ is a contracted chain complex. If the pseudo-complex was already a complex, $\partial_{*}^{\prime}=\hat{\partial}_{*}$, and if in addition $s_{*}$ was already a contraction, then $s_{*}^{\prime}=\hat{s}_{*}$.

Proof. The proof follows the same outline as for Proposition 6.4, but we notice that the map $\psi_{r}=1_{C_{r}}+u$ has the additional property that $u^{i+1}=0$ on the quotient $C_{r} / F_{i} C_{r}$. This follows by induction from the exact sequences

$$
0 \rightarrow F_{i+1} C / F_{i} C \rightarrow F_{0} C / F_{i+1} C \rightarrow F_{0} C / F_{i} C \rightarrow 0
$$

of pseudo-contractible complexes. Then $\psi_{r}$ induces an invertible map on $C_{r} / F_{i} C_{r}$, for each $i \geq 0$. We define $s_{r}^{\prime}=s_{r} \circ \psi_{r}^{-1}$ on $C_{r} / F_{i} C_{r}$ as before. By induction, we have constructed contraction data $\left(C / F_{i} C, \partial^{\prime}, s^{\prime}\right)$, for each $i \geq 0$. In addition, this contraction data is compatible with the projections $C / F_{i+1} C \rightarrow C / F_{i}$, and hence induce contraction data $\left(\widehat{C}, \partial^{\prime}, s^{\prime}\right)$ for the inverse limit complex.

Remark 6.7. Once again, this process doesn't change $\partial_{1}$, so the new contractible complex gives an expression for $\widehat{C}_{0}$ as a direct summand of $\widehat{C}_{1}$, with respect to completion of the original boundary map $\partial_{1}: C_{1} \rightarrow C_{0}$.

Example 6.8 (Whitehead groups). Define the Whitehead groups, $W h_{n}(\mathbf{Z} G)$, as the homotopy groups of the spectrum which is the cofibre of the Loday assembly map

$$
B G^{+} \wedge K(\mathbf{Z}) \rightarrow K(\mathbf{Z} G)
$$

The Loday assembly map is a map of bifunctors [Nicas 1987, Main Theorem, page 223], and the Whitehead groups are bifunctors. Furthermore, the $W h_{n}, n \leq$ 3, are Mackey functors, but it is not obvious from this description that the other higher Whitehead groups are actually Mackey functors. However, from the long exact sequence in homotopy theory we see that they are pseudo-Mackey functors. From Example 3.12, Example 5.10 and Proposition 6.4, we see that the $W h_{n}(\mathbf{Z G )}$ are computed by the hyperelementary family. Similarly, the $W h_{n}(\mathbf{Z} G) \otimes \mathbf{Z}_{(p)}$ are 
computed by the $p$-hyperelementary family. See [Swan 1970; Lam 1968; Nicas 1987] for partial results in this direction).

Example 6.9 (Tate cohomology). The Tate cohomology of $W h_{n}$ or Quillen's $K_{n}$ are bifunctors which are subquotients of $W h_{n}$ or $K_{n}$, and hence are computed by the hyperelementary family. The localization maps $W h_{n} \rightarrow W h_{n} \otimes \mathbf{Z}_{(2)}$ and $K_{n} \rightarrow K_{n} \otimes \mathbf{Z}_{(2)}$ induce isomorphisms on Tate cohomology. Hence the Tate cohomology is computed by the 2-hyperelementary family. Given any pseudo-Mackey subfunctor of $W h_{n}$ or $K_{n}$ which is invariant under the involution, we can form the Tate cohomology and this Tate cohomology functor is computed by any family which contains the 2-hyperelementary family.

\section{Surgery obstruction groups}

Dress [1975, Theorem 1] claims computability results for "any of the $L$-functors defined by C. T. C. Wall" (in [Wall 1976]). However, the nonoriented $L$-groups $L_{n}(\mathbf{Z} G, \omega)$ are not always Mackey functors, and so the techniques described in [Dress 1975] do not appear to be adequate to prove the result in this generality. The point is that an inner automorphism by an element $g \in G$ with $\omega(g)=-1$ induces multiplication by -1 (which may not be the identity) on $L_{n}(\mathbf{Z} G, \omega)$ [Taylor 1973]. One of the main applications of our more general techniques is to supply a proof that nonoriented $L$-theory is hyperelementary computable, in the sense that $L_{n}(\mathbf{Z} G, \omega)$ is the limit of restrictions or inductions involving hyperelementary subgroups of $G$.

Fix a finite group $G$, and the geometric antistructure for which $\theta=\mathrm{id}$ and $b=e \in$ $G$ [HTW 1990, 1.B.3]. Let $\omega: G \rightarrow\{ \pm 1\}$ be a fixed orientation homomorphism, and for each subgroup $H \subset G$ let $\omega_{H}=\left.\omega\right|_{H}$. We define the following categories:

(1) $\mathbf{A}(G, \omega)$, with objects finite groups $H$ isomorphic to some subgroup of $G$, and morphisms given by a Grothendieck group construction on finite biset forms $\left(X, \omega_{X}\right)$ (see [HTW 1990, page 256] for the definition). We construct A. $(G, \omega)$ by taking the additive completion.

(2) $(R,-)$-Morita, with objects and morphisms as defined in [HTW 1990, 1.B.2], and the quotient category $(R,-)$-Witt from [HTW 1990, 1.C.2], for any commutative ring $R$ with unit.

(3) $(R G, \omega)$-Morita, with objects $H$ isomorphic to some subquotient $K / N$ of $G$, with $N \subset \operatorname{ker} \omega$, and morphisms given by the Grothendieck group construction on finite biset forms $\left(X, \omega_{X}\right)$, modulo an equivalence relation, as defined in [HTW 1990, 1.B.3]. We can define the analogous quotient category $(R G, \omega)$-Witt by setting metabolic forms to zero in the morphisms [HTW 1990, page 254]. 
Notice that by forgetting the orientation map $\omega$ we get functors into the categories discussed in Section 5. The construction of Definition 5.4 gives a prebifunctor

$$
j: \mathscr{D}(G) \rightarrow \mathbf{A} \bullet(G, \omega)
$$

extending the prefunctor $\operatorname{Or}(G) \rightarrow \mathbf{A} .(G, \omega)$ out of the orbit category, defined on objects by $G / H \mapsto H$ and on morphisms by sending the $G$-map $f: G / H \rightarrow G / K$, given by $f(e H)=g K$, to the biset form $\left({ }_{K} K_{g^{-1} K g}, \omega_{K}\right)$. This definition depends on the choice of coset representative $g$ for the morphism $f$ in $\operatorname{Or}(G)$, since this time, if $x \in K$ and $\omega(x)=-1$, the two morphisms $e H \mapsto g K$ and $e H \mapsto g x K$ are sent to different biset forms.

Lemma 7.1. In A . $(G, \omega)$, the morphism $\left[{ }_{H} H_{x^{-1} H x}, \omega_{H}\right]=\omega(x) \cdot$ id for all $x \in H$. If $F: \mathbf{A} .(G, \omega) \rightarrow A b$ is an additive functor, then

$$
F \circ j: \mathbf{A} \cdot(G, \omega) \rightarrow A b
$$

is a Mackey prefunctor, which is a Mackey functor if and only if all the inner automorphism morphisms $F\left({ }_{H} H_{x^{-1} H x}, \omega_{H}\right)=\mathrm{id}$, for all $x \in H$.

Proof. The identity morphism in A. $(G, \omega)$ is represented by the biset form

$$
\left({ }_{H} H_{H}, \omega_{H}\right) \text {. }
$$

The map $\psi:{ }_{H} H_{H} \rightarrow{ }_{H} H_{x H x^{-1}}$ of biset forms defined by $\psi(h)=h x^{-1}$, see (5.3), induces an isometry of biset forms $\left({ }_{H} H_{x^{-1} H x}, \omega_{H}\right) \cong\left({ }_{H} H_{H}, \omega(x) \cdot \omega_{H}\right)$ and hence

$$
\left[{ }_{H} H_{x^{-1} H x}, \omega_{H}\right]=\omega(x) \cdot \mathrm{id}
$$

in the Grothendieck group of morphisms of $\mathbf{A} .(G, \omega)$.

The property (M1) depends on conjugations acting trivially, or in other words, should induce $F(\psi)=$ id for all $x \in H$ (including those with $\omega(x)=-1$ ).

The $R$-group ring functor of [HTW 1990, 1.B.4] induces a functor from A . $(G, \omega)$ to $(R G, \omega)$-Morita or further into $(R,-)$-Witt. The required formulas are in section 1.B of [HTW 1990], including the remark that since our morphisms are formed via a Grothendieck construction, we are entitled to equate metabolics on isomorphic modules. There is a functor $a: \mathbf{A} .(G, \omega) \rightarrow(R G, \omega)$-Morita as before, and we let

$$
d: \mathscr{D}(G) \rightarrow(R G, \omega) \text {-Morita }
$$

be the prebifunctor $d=a \circ j$. There is a homomorphism from the Dress ring

$$
G U(H, \mathbf{Z}) \rightarrow \operatorname{Hom}_{(R,-) \text {-Morita }}(H, H)
$$

given by tensor product (see [Dress 1975] where it is asserted that $G U(G, \mathbf{Z})$ acts on $L$-theory, or [Hambleton, Ranicki and Taylor 1987, page 143] for explicit formulas). Dress [1975] showed that the hyperelementary family contracts the 
Dress ring. We observe that the same formulas give an action of the Burnside quotient Green ring $\mathscr{A}_{G U}$ on $(R G, \omega)$-Morita.

Theorem 7.2. Let $F:(R G, \omega)$-Morita $\rightarrow A$ Ab be an additive functor. Then

$$
F \circ d: \mathscr{D}(G) \rightarrow A d b
$$

is a Mackey prefunctor, and the 2-adic completion of any such Mackey prefunctor is 2-hyperelementary computable. If $\mathcal{M}=F \circ d$ is a Mackey functor, then $\mathcal{M}$ is hyperelementary computable.

Proof. In the oriented case $(\omega \equiv 1)$ the prefunctor $\mathcal{M}$ is actually a Mackey functor, by Lemma 7.1. More generally, whenever $M=F \circ d$ is a Mackey functor the result follows as in Theorem 5.5, since $\mathcal{M}$ is a Green module over $\mathscr{A}_{G U}$. By [Dress 1975, Theorem 3], and Theorem 3.6, the Burnside quotient Green ring of the Dress ring is hyperelementary computable.

In the nonoriented case, we define a filtration $F_{i}=2^{i} F, i \geq 0$, with $F_{0}=F$, and note that the subquotients $\left(F_{i} / F_{i+1}\right) \circ d$ are Mackey functors. Now we let $(C, \partial)$ denote the filtered Amitsur pseudo-complex for $F_{*} \circ d$ with respect to 2hyperelementary induction, and the result follows from Proposition 6.6. Notice that the passage from a pseudo-contractible pseudo-complex to a contractible complex does not change the first boundary map, so $F \circ d$ is 2-adically detected (generated) by the given restriction (induction) maps to the 2-hyperelementary subgroups.

Example 7.3 (Nonoriented $L$-theory). The main example for us is the surgery obstruction group $L_{n}(\mathbf{Z G}, \omega)$. It is a foundational result of Wall [1974] that the surgery obstruction groups for finite groups are finitely-generated, with 2-primary torsion exponent. Theorem 7.2 computes $L_{n}(\mathbf{Z} G, \omega) \otimes \widehat{\mathbf{Z}}_{2}$ as a limit (and as a colimit) over the 2-hyperelementary subgroups $H \subset G, H \in \mathscr{H}$. These limits use the standard induction or restriction maps, for example, for induction we have the surjective map

$$
\partial_{1}: \bigoplus_{H \in \mathscr{H}} L_{n}(\mathbf{Z} H, \omega) \otimes \widehat{\mathbf{Z}}_{2} \rightarrow L_{n}(\mathbf{Z} G, \omega) \otimes \widehat{\mathbf{Z}}_{2}
$$

and our contraction data gives the relation subgroup $\operatorname{ker} \partial_{1}=\operatorname{Im} \partial_{2}^{\prime}$.

We conclude that $L_{n}(\mathbf{Z} G, \omega)$ is also effectively 2-hyperelementary computable: the torsion subgroup is isomorphic to that of $L_{n}(\mathbf{Z} G, \omega) \otimes \widehat{\mathbf{Z}}_{2}$, and the divisibility of the signatures is computable since the kernel and cokernel of the natural transformation

$$
L_{n}(\mathbf{Z} G, \omega) \rightarrow L_{n}(\mathbb{R} G, \omega)
$$

of pseudo-Mackey functors are both 2-primary torsion groups [Wall 1974, 7.3, 7.4]. The groups $L_{n}(\mathbb{R} G, \omega)$ were computed explicitly in [Wall 1976, 2.2.1] in terms of the irreducible characters of $G$. The proof of computability given here applies in 
the oriented case $(\omega \equiv 1)$, but in that case the $L$-group is a Mackey functor and the argument is essentially the same as the one given by Dress. Other important examples were listed in [HTW 1990, 1.B.8].

Example 7.4 ( $L$-theory with decorations). Let $R$ be a commutative ring with unit, and consider any $L$-group $L_{n}^{B}(R G, \omega)$ for $R G$ with antiinvolution given by $\omega$ : $G \rightarrow\{ \pm 1\}$ with decoration in any involution invariant subbifunctor, $B$, of $K_{i}(\mathbf{Z} G)$ or $W h_{i}(\mathbf{Z} G), i \leq 1$; see [Hambleton, Ranicki and Taylor 1987] for a summary of the definitions. It was checked in Theorem 5.3, Corollary 5.5 and Example 5.14 of that same reference that the corresponding round $L$-theories are functors out of $(\mathbf{Z} G, \omega)$-Morita. Hence these $L$-theories are pseudo-Mackey functors and are contracted by the hyperelementary family. It was also checked in Proposition 5.6, Corollary 5.7 and Example 5.14 of the same paper that the corresponding ordinary $L$-theories are functors out of $(\mathbf{Z} G, \omega)$-Morita, so the same computation result holds.

Example 7.5 (Localization). Dress [1975] shows that the Dress ring $G U$ is contracted by any family containing the 2-hyperelementary and $p$-elementary families. More precisely, he showed that the 2-localization of the Dress ring is contracted by the 2-hyperelementary family, and the $p$-localization, $p$ odd, is contracted by the $p$-elementary family.

Proposition 6.4 and a standard mixing argument shows that this smaller family suffices to contract the $L^{B}$ functors described above. For subbifunctors $B$ closed under the action of the Dress ring, this was proved by Dress [1975] and Wall [1976]. A similar argument shows that the odd-dimensional $L^{B}$-groups are contracted by the 2-hyperelementary family alone.

Example 7.6 (Symmetric, hyper-quadratic and lower $L$-theory). The Ranicki symmetric and hyper-quadratic $L$-theories [Ranicki 1992] are also functors out of $(\mathbf{Z} G, \omega)$-Morita and hence are contracted by the hyperelementary family. The hyper-quadratic theory is a 2-torsion group with an exponent so it is contracted by the 2-hyperelementary family (as above, we note that the 2-localization map induces an isomorphism on this functor and use the 2-local contraction of functors out of $(\mathbf{Z},-)$-Morita by the 2-hyperelementary family). The lower $L$-theories for a ring with antistructure can be defined in terms of the $L$-theory of the ring with some Laurent variables adjoined [Ranicki 1992] and hence are functors out of $(\mathbf{Z} G, \omega)$ Morita. Therefore $L$-theories with decorations in sub-Mackey functors of $K_{i}$ for $i<0$ are contracted by the hyperelementary family. The higher $L$-theories of Weiss and Williams [1989] should also be amenable to these techniques.

Example 7.7 (Farrell-Hsiang induction). The technique of Farrell and Hsiang [1977, §§1-2] was originally introduced to apply induction theory to the $L$-groups of an infinite group $\Gamma$. Let $\mathrm{pr}: \Gamma \rightarrow G$ be a homomorphism to a finite group 
$G$, and define an orientation character for $\Gamma$ by the composition $\omega \circ \mathrm{pr}$, where $\omega: G \rightarrow\{ \pm 1\}$ is an orientation character for $G$. Then $L_{*}^{B}(R \Gamma, \omega)$ is an additive functor $(R G, \omega)$-Witt $\rightarrow \mathscr{A} b$, which defines a pseudo-Mackey functor as above. To check this, note that we again have a generating set for the morphisms consisting of the bisets $X=H_{2} \times_{K} H_{1}$, where $K \subset H_{2} \times H_{1}$ is a subgroup. To produce the needed biform on $X$, we adapt the formulas in [HTW 1990, 1.B] with $\theta_{X}=$ id. If $\omega \equiv 1$, it follows that these $L$-groups can be computed in terms of the $L$-theory of the various subgroups $\Gamma_{H}=\operatorname{pr}^{-1}(H), H \subset G$. In particular, it is enough to use the hyperelementary subgroups $H$ of $G$.

\section{Acknowledgement}

We were greatly indebted to L. Gaunce Lewis, Jr. for his meticulous reading of our 1990 MSRI preprint [Hambleton et al. 1990], and for his extensive helpful comments. He pointed out to us that the definition of the Burnside quotient Green ring appears in his unpublished notes, The theory of Green functors (1980). This paper is dedicated to his memory.

\section{References}

[Bak 1995] A. Bak, "Induction for finite groups revisited", J. Pure Appl. Algebra 104:3 (1995), 235-241. MR 96m:19002 Zbl 0844.19001

[Bouc 2007] S. Bouc, "The functor of units of Burnside rings for p-groups", Comment. Math. Helv. 82:3 (2007), 583-615. MR 2008e:19001 Zbl 1142.19001

[Bouc 2008] S. Bouc, "Rational p-biset functors", J. Algebra 319:4 (2008), 1776-1800. MR 2009a: 20032 Zbl 1149.19001

[tom Dieck 1987] T. tom Dieck, Transformation groups, de Gruyter Studies in Mathematics 8, Walter de Gruyter \& Co., Berlin, 1987. MR 89c:57048 Zbl 0611.57002

[Dress 1973] A. W. M. Dress, "Contributions to the theory of induced representations", pp. 183-240 in Algebraic K-theory, II: "Classical" algebraic K-theory and connections with arithmetic, edited by H. Bass, Lecture Notes in Math. 342, Springer, Berlin, 1973. MR 52 \#5787 Zbl 0331.18016

[Dress 1975] A. W. M. Dress, "Induction and structure theorems for orthogonal representations of finite groups", Ann. of Math. (2) 102:2 (1975), 291-325. MR 52 \#8235 Zbl 0315.20007

[Farrell and Hsiang 1977] F. T. Farrell and W. C. Hsiang, "Rational L-groups of Bieberbach groups", Comment. Math. Helv. 52:1 (1977), 89-109. MR 56 \#6679 Zbl 0364.57003

[Farrell and Hsiang 1981] F. T. Farrell and W. C. Hsiang, "The Whitehead group of poly-(finite or cyclic) groups", J. London Math. Soc. (2) 24:2 (1981), 308-324. MR 83b:20041 Zbl 0514.57002

[Green 1971] J. A. Green, "Axiomatic representation theory for finite groups", J. Pure Appl. Algebra 1:1 (1971), 41-77. MR 43 \#4931 Zbl 0249.20005

[Hambleton 2006] I. Hambleton, "Some examples of free actions on products of spheres", Topology 45:4 (2006), 735-749. MR 2007b:57065 Zbl 1099.57028

[Hambleton and Lück 2007] I. Hambleton and W. Lück, "Induction and computation of Bass nil groups for finite groups", preprint, 2007. arXiv arXiv:0706.4019v1 
[Hambleton and Pedersen 1991] I. Hambleton and E. K. Pedersen, "Bounded surgery and dihedral group actions on spheres", J. Amer. Math. Soc. 4 (1991), 105-126. MR 91h:57022 Zbl 0727.57034

[Hambleton and Pedersen 2004] I. Hambleton and E. K. Pedersen, "Identifying assembly maps in K - and L-theory”, Math. Ann. 328:1-2 (2004), 27-57. MR 2004j:19001 Zbl 1051.19002

[Hambleton and Taylor 1999] I. Hambleton and L. R. Taylor, "Rational permutation modules for finite groups", Math. Z. 231:4 (1999), 707-726. MR 2000i:20013 Zbl 0926.20009

[Hambleton and Taylor 2000] I. Hambleton and L. R. Taylor, "A guide to the calculation of the surgery obstruction groups for finite groups", pp. 225-274 in Surveys on surgery theory, vol. 1, edited by S. Cappell et al., Ann. of Math. Stud. 145, Princeton Univ. Press, Princeton, NJ, 2000. MR 2001e:19007 Zbl 0952.57009

[Hambleton et al. 1990] I. Hambleton, L. R. Taylor, and B. Williams, "Induction theory", MSRI Preprint 05425-90, 1990.

[Hambleton, Ranicki and Taylor 1987] I. Hambleton, A. Ranicki, and L. R. Taylor, "Round Ltheory", J. Pure Appl. Algebra 47:2 (1987), 131-154. MR 88i:18010 Zbl 0638.18003

[HTW 1990] I. Hambleton, L. R. Taylor, and B. Williams, "Detection theorems for $K$-theory and L-theory”, J. Pure Appl. Algebra 63:3 (1990), 247-299. MR 91b:18015 Zbl 0718.18006

[HTW 2008] I. Hambleton, L. R. Taylor, and B. Williams, "Mackey functors and bisets", preprint, 2008. arXiv 0806.4054

[Lam 1968] T.-y. Lam, "Induction theorems for Grothendieck groups and Whitehead groups of finite groups”, Ann. Sci. École Norm. Sup. (4) 1 (1968), 91-148. MR 38 \#217 Zbl 0164.02703

[Lindner 1976] H. Lindner, "A remark on Mackey-functors", Manuscripta Math. 18:3 (1976), 273278. MR 53 \#5691 Zbl 0321.18002

[MacLane 1971] S. MacLane, Categories for the working mathematician, 2nd ed., Graduate Texts in Mathematics 5, Springer, New York, 1971. MR 50 \#7275 Zbl 0705.18001

[Nicas 1987] A. J. Nicas, "Frobenius induction for higher Whitehead groups", Canad. J. Math. 39:1 (1987), 222-238. MR 88k:55013 Zbl 0637.18006

[Oliver 1988] R. Oliver, Whitehead groups of finite groups, London Mathematical Society Lecture Note Series 132, Cambridge University Press, 1988. MR 89h:18014 Zbl 0636.18001

[Ranicki 1992] A. Ranicki, Lower $K$ - and L-theory, London Mathematical Society Lecture Note Series 178, Cambridge University Press, 1992. MR 94f:19001 Zbl 0752.57002

[Swan 1970] R. G. Swan, K-theory of finite groups and orders, Lecture Notes in Mathematics 149, Springer, Berlin, 1970. MR 46 \#7310 Zbl 0205.32105

[Taylor 1973] L. R. Taylor, "Surgery groups and inner automorphisms”, pp. 471-477 in Algebraic K-theory, III: Hermitian K-theory and geometric applications, edited by H. Bass, Lecture Notes in Math. 343, Springer, Berlin, 1973. MR 53 \#9253 Zbl 0267.18017

[Thévenaz 1990] J. Thévenaz, "A visit to the kingdom of the Mackey functors", pp. 215-241 in Darstellungstheorietage (Sion, Switzerland, 1989), Bayreuth. Math. Schr. 33, 1990. MR 91c:20022 Zbl 0703.20006

[Thévenaz and Webb 1995] J. Thévenaz and P. Webb, "The structure of Mackey functors", Trans. Amer. Math. Soc. 347:6 (1995), 1865-1961. MR 95i:20018 Zbl 0834.20011

[Wall 1974] C. T. C. Wall, "On the classification of Hermitian forms. V. Global rings", Invent. Math. 23 (1974), 261-288. MR 54 \#12861 Zbl 0278.16018

[Wall 1976] C. T. C. Wall, "Classification of Hermitian Forms. VI. Group rings”, Ann. of Math. (2) 103:1 (1976), 1-80. MR 55 \#5720 Zbl 0328.18006 
[Wall 1999] C. T. C. Wall, Surgery on compact manifolds, 2nd ed., edited by A. A. Ranicki, Mathematical Surveys and Monographs 69, American Mathematical Society, Providence, RI, 1999. MR 2000a:57089 Zbl 0935.57003

[Weiss and Williams 1989] M. Weiss and B. Williams, "Automorphisms of manifolds and algebraic K-theory. II”, J. Pure Appl. Algebra 62:1 (1989), 47-107. MR 91e:57055 Zbl 0691.57012

Communicated by Dave Benson

Received 2008-03-27 Revised 2009-05-01 Accepted 2009-05-04

hambleton@mcmaster.ca McMaster University, Department of Mathematics \& Statistics, Hamilton, ON L8S 4K1, Canada

taylor.2@nd.edu University of Notre Dame, Department of Mathematics, Notre Dame, IN 46556, United States

williams.4@nd.edu University of Notre Dame, Department of Mathematics, Notre Dame, IN 46556, United States 